\title{
Article \\ Variation in Parasitoid Virulence of Tetrastichus brontispae during the Targeting of Two Host Beetles
}

\author{
Hua-Jian Zhang 1,2, Ya-Ping Lin 1,2, Hong-Yu Li 1,2, Rui Wang 1,2, Lang Fu 1,2, Qing-Chen Jia 1,2, \\ You-Ming Hou $1,2, *$ (D) and Bao-Zhen Tang $1,2, *$ (D) \\ 1 State Key Laboratory of Ecological Pest Control for Fujian and Taiwan Crops, Fujian Agriculture and \\ Forestry University, Fuzhou 350002, China; huajianzhang@fafu.edu.cn (H.-J.Z.); \\ linyaping@fafu.edu.cn (Y.-P.L.); snow_lhy@163.com (H.-Y.L.); wangrui@fafu.edu.cn (R.W.); \\ m18305918768@163.com (L.F.); jiaqingchen@fafu.edu.cn (Q.-C.J.) \\ 2 Key Lab of Biopesticide and Chemical Biology, Ministry of Education \& Fujian Provincial Key Laboratory of \\ Insect Ecology, Department of Plant Protection, Fujian Agriculture and Forestry University, \\ Fuzhou 350002, China \\ * Correspondence: ymhou@fafu.edu.cn (Y.-M.H.); tangbaozhen@fafu.edu.cn (B.-Z.T.)
}

Citation: Zhang, H.-J.; Lin, Y.-P.; Li, H.-Y.; Wang, R.; Fu, L.; Jia, Q.-C.; Hou, Y.-M.; Tang, B.-Z. Variation in Parasitoid Virulence of Tetrastichus brontispae during the Targeting of Two Host Beetles. Int. J. Mol. Sci. 2021, 22, 3581. https://doi.org/10.3390/ ijms22073581

Academic Editor: Klaus H. Hoffmann

Received: 8 February 2021

Accepted: 27 March 2021

Published: 30 March 2021

Publisher's Note: MDPI stays neutral with regard to jurisdictional claims in published maps and institutional affiliations.

Copyright: (c) 2021 by the authors. Licensee MDPI, Basel, Switzerland. This article is an open access article distributed under the terms and conditions of the Creative Commons Attribution (CC BY) license (https:/ / creativecommons.org/licenses/by/ $4.0 /)$.

\begin{abstract}
In host-parasitoid interactions, antagonistic relationship drives parasitoids to vary in virulence in facing different hosts, which makes these systems excellent models for stress-induced evolutionary studies. Venom compositions varied between two strains of Tetrastichus brontispae, $\mathrm{Tb}-\mathrm{Bl}$ and $\mathrm{Tb}-\mathrm{On}$. Tb-Bl targets Brontispa longissima pupae as hosts, and $\mathrm{Tb}-\mathrm{On}$ is a sub-population of $\mathrm{Tb}-\mathrm{Bl}$, which has been experimentally adapted to a new host, Octodonta nipae. Aiming to examine variation in parasitoid virulence of the two strains toward two hosts, we used reciprocal injection experiments to compare effect of venom/ovarian fluids from the two strains on cytotoxicity, inhibition of immunity and fat body lysis of the two hosts. We found that Tb-Onvenom was more virulent towards plasmatocyte spreading, granulocyte function and phenoloxidase activity than Tb-Blvenom. Tb-Blovary was able to suppress encapsulation and phagocytosis in both hosts; however, Tb-Onovary inhibition targeted only B. longissima. Our data suggest that the venom undergoes rapid evolution when facing different hosts, and that the wasp has good evolutionary plasticity.
\end{abstract}

Keywords: adaptive evolution; host selection; immunosuppressive; parasitic adaptability; parasitic strategy

\section{Introduction}

Parasitoid wasps represent $10 \%$ to $20 \%$ of all insects, and they are the most successful and important groups of natural enemies used as biological control agents for insect pests [1-3]. Wasps take advantage of a variety of strategies to subdue the hosts to generate a comfortable environment for the development of their offspring, usually inducing the impairment of the host insect [4]. Endoparasitoids develop inside their hosts; thus, they must evade or counteract the specific physiological and immunological defences of their hosts [2,5,6]. Generally, in the host-parasitoid interactions, the antagonistic relationships drives endoparasitoids to evolve to become more virulent when encountering hosts with stronger selection pressure, which contributes to biological diversity and makes these systems excellent models for stress-induced evolutionary studies [2,7-9]. In this evolutionary race, strategies used by wasp parasitoids are mainly divided into the active and passive mechanisms. For the active mechanisms, endoparasitoids adopt a number of virulence factors, including virus-like particles (VLPs), polydnaviruses (PDVs), ovarian fluids, venom and teratocytes, to disrupt the host immune system directly or indirectly (direct intervention) and help them to escape the host encapsulation and melanisation [10-16]. In the case of the passive mechanisms, endoparasitoids lay their eggs in a special tissue or at a special life stage of the hosts with a low integrated immunity system to avoid 
encapsulation, or they depend on their inherent surface features that will mislead the host immunity to disable the recognition of the parasitoid as non-self and avoid triggering an immune induction (passive dodge) [10,17-21].

Parasitoid tracking of locally common host genotypes results in local adaptation [9,22]. However, upon encountering an alternative host, the parasitoid may miss an opportunity to interact with its inherent targets generated by a long-term evolution from the native host and bring about excessive energy cost [23-27]; thus, the variability of virulence is required. Variations of parasitoid virulence can originate from two main mechanisms; either they differ in their ability to locally evade the host immune system or they differ in their abilities to suppress the whole encapsulation response. To balance the costs in development of a higher virulence, parasitoids tend to vary in their virulence towards different hosts and do not evolve high infectivity in all hosts [28]. For example, in the Asecodes parviclava (Hymenoptera: Eulophidae) and Galerucella system, the differences in the immune defence among G. pusilla, G. tenella and G. calmariensis from different areas result in different virulent strains of A. parviclava; the wasps emerged from G. pusilla can favourably infect the larvae of all three species, and the wasps emerged from G. tenella may also successfully infect the larvae of G. tenella and G. calmariensis; nevertheless, the parasitoids from G. calmariensis can only infect the larvae of G. calmariensis [9]. Evidently, the successful parasitism of A. parviclava in different hosts is deeply influenced by the native host species, i.e., host species and geographic variations promote the parasitoid trade-off in virulence due to differences in the immune defence, which will finally lead to variations in the host range [9,29]. In the Aphelinus certus and Aphis system, the genetic differentiation of Aphis populations results in inter-population differences in parasitism, indicating that differential host specificity of the parasitoid populations is influenced by genetic distances of the hosts [30].

Variations of virulence in alternative hosts can be driven by a gene-for-gene matching, which may lead to evolution of different genes wasps allowing their adaptation to different hosts $[9,29,31,32]$. Notably, venom genes can evolve depending on the variation of encountered stress. For example, in the Leptopilina boulardi and Drosophila system, the injection of venom from the ISm and ISy strains to D. melanogaster and D. yakuba, respectively, may generate virulence by incapacitating the encapsulation reaction to a foreign body. In contrast, the injection of venom from the avirulent line (ISm for D. yakuba and ISy for D. melanogaster) does not have any influence on the encapsulation abilities of the hosts [33]. These results indicate that the qualitative changes in the venoms of different parasitoid lines are responsible for the variations in the parasitoid virulence $[12,23,34]$. Venoms contain proteins that function by altering host development [4], nutrient flux [35-37], immune defence [38-40] and lipid metabolism [41,42], and they have been confirmed to participate in multiple evolutionary processes [43-45], such as co-option, gene duplication, alternative splicing, multi-functionalisation, horizontal gene transfer and de novo gene synthesis [44,46-48]. Excitingly, in recent years, the whole venom proteomic analysis allowed the investigation of the multifunctional and evolutionary processes of the venom proteins from a comprehensive perspective and the study of the mechanisms of the evolution of countermeasures to different hosts. The gene-by-gene approach has provided additional details about the functions of the venom proteins and the interactions between the wasps and their hosts. However, studies of the parasitoid biology remain mysterious, and additional work has to be invested in the host-parasitoid interactions.

Tetrastichus brontispae (Eulophidae) is native to Java (Indonesia) and is a gregarious and idiobiont endoparasitoid that has been used to efficiently control two Chrysomelidae beetles, namely, Brontispa longissima (Gestro) and Octodonta nipae (Maulik), which are dangerous invasive pests of the palm plants in southern China $[49,50]$. T. brontispae has certain favourable characteristics that enhance its success to control the two beetles, including approximately 20 days for the whole lifecycle, preference for fresh host pupae, and high fecundity, with approximately 22 wasps emerging from each host [49,50]. T. brontispae lacks polydnavirus and virus-like filaments and thus, venom is recognised as its main 
virulence factor [21,51]. Proteomics and transcriptomics enable to investigate the venom compositions at the molecular level, thereby promoting the studies of the parasitoid biology of T. brontispae [51]. Previous comparative proteome analyses have shown that venom compositions varied between the two strains, T. brontispae- $\mathrm{Bl}(\mathrm{Tb}-\mathrm{Bl})$ and T. brontispae- $\mathrm{On}$ ( $\mathrm{Tb}$-On), even though the major venom proteins were shared between the two strains [51]. The Tb-Bl strain targets B. longissima pupae as hosts, and the Tb-On strain is a derived sub-population of $\mathrm{Tb}-\mathrm{Bl}$, which has been experimentally adapted to a new host, O. nipae, for six years (120 generations). The functions of the venom and ovarian fluid of $T$. brontispae differ in the case of successful parasitism [21]. Therefore, in this study, the virulence of the venoms ( $\mathrm{Tb}$-Onvenom and $\mathrm{Tb}$-Blvenom) and ovarian fluids (Tb-Onovary and $\mathrm{Tb}$-Blovary) from the two strains towards the hosts were examined by reciprocal transplant style experiments aiming to explore whether the venoms and ovarian fluids from the two strains have differences in the virulence activities towards the two hosts, and confirm stress-induced evolutionary events in the parasitoid wasp. Based on previous studies on the same topic, we hypothesise that $\mathrm{Tb}$-Onvenom and $\mathrm{Tb}$-Blvenom will show different virulent activities in regulation host immunity, which helps T. brontispae generate adaptation in B. longissima and O. nipae.

\section{Results}

2.1. Virulence of Venom and Ovarian Fluids against Host Total Haemocyte Counts (THCs) and Differential Haemocyte Counts (DHCs)

In both un-stung hosts, the general cell composition included prohaemocytes, granulocytes, plasmatocytes, spherulocytes and oenocytoids; plasmatocytes and granulocytes were the most abundant (over 70\%) (Figure S1A). Notably, granulocytes with multiple morphology were common in the two hosts (Figure S1B). THCs in O. nipae was substantially higher than that in B. longissima ( $24 \mathrm{~h}, t=4.302, \mathrm{df}=56, p<0.0001$ ) (Figure S1C). After injections of PBS/venom/ovarian fluids, THCs were increased over time in both hosts, peaking at $48 \mathrm{~h}$ for venom/ovarian fluids and at $72 \mathrm{~h}$ for PBS in B. longissima (Figure S1D; PBS, $F_{(4,145)}=4.672, p<0.01$; Tb-Blvenom, $F_{(4,140)}=2.955, p<0.05 ;$ Tb-Blovary, $F_{(4,145)}=2.853$, $p<0.05$ ) and peaking at $96 \mathrm{~h}$ for all samples in $O$. nipae (Figure S1E; PBS, $F_{(4,142)}=5.458$, $p<0.001$; Tb-Onvenom, $F_{(4,147)}=4.871, p=0.001$; Tb-Onovary, $\left.F_{(4,152)}=11.66, p<0.001\right)$. The injection of ovarian fluids had no influence on THCs compared with THCs detected after PBS injections in both hosts at each time point (Figure S1D,E) except that at $96 \mathrm{~h}$ post injection of Tb-Onovary, the THCs in O. nipae was significantly decreased (Figure S1F; $\left.96 \mathrm{~h}, F_{(2,84)}=13.6, p<0.0001\right)$. The injection of Tb-Onvenom induced a decrease in THCs in O. nipae at 24, 48 and $96 \mathrm{~h}$ compared with that in the PBS group (Figure S1E,F; $24 \mathrm{~h}$, $\left.F_{(2,87)}=4.413, p<0.05 ; 48 \mathrm{~h}, F_{(2,105)}=12.47, p<0.0001 ; 96 \mathrm{~h}, F_{(2,84)}=13.6, p<0.0001\right)$.

To investigate whether mother's host experience influenced the virulence of venom and ovarian fluids, $\mathrm{Tb}$-Onvenom and $\mathrm{Tb}$-Onovary were injected into B. longissima, and $\mathrm{Tb}$ Blvenom and $\mathrm{Tb}$-Blovary were injected into $O$. nipae. The reciprocal virulent influence was evaluated at $24 \mathrm{~h}$ post injection. The results indicate that in B. longissima pupae, the injection of Tb-Onvenom, Tb-Onovary, Tb-Blvenom and Tb-Blovary had no influence on THCs, while in $O$. nipae pupae, the injection of Tb-Onvenom or Tb-Blvenom induced a significant decrease in THCs $\left(F_{(4,116 \cdot 6)}=3.710, p<0.01 ; W_{(4,70.05)}=3.139, p=0.0196\right)$ (Figure 1A,B). In the case of DHCs, the ratio of plasmatocytes to granulocytes was approximately 1:1.2 in O. nipae and 1.5:1 in B. longissima (Figure 1C), respectively, after PBS injection. In $B$. longissima, macrogranulocytes were significantly increased after challenge by virulent factors (Figure 1C), especially by Tb-Onvenom (12.45\%) and Tb-Blvenom (15.7\%), compared to $4.68 \%$ detected in the case of PBS $\left(\chi^{2}=62.255, \mathrm{df}=16, p<0.0001\right)$. In O. nipae, the injection of venom or ovarian fluids from both strains markedly influenced DHCs (Figure 1C; $\chi^{2}=128.538, \mathrm{df}=16, p<0.0001$ ) and induced a decrease in granulocytes by $19 \%$ (dark green section), an increase in plasmatocytes by $15 \%$ (pink section) and an increase in macrogranulocytes by $8 \%$. 


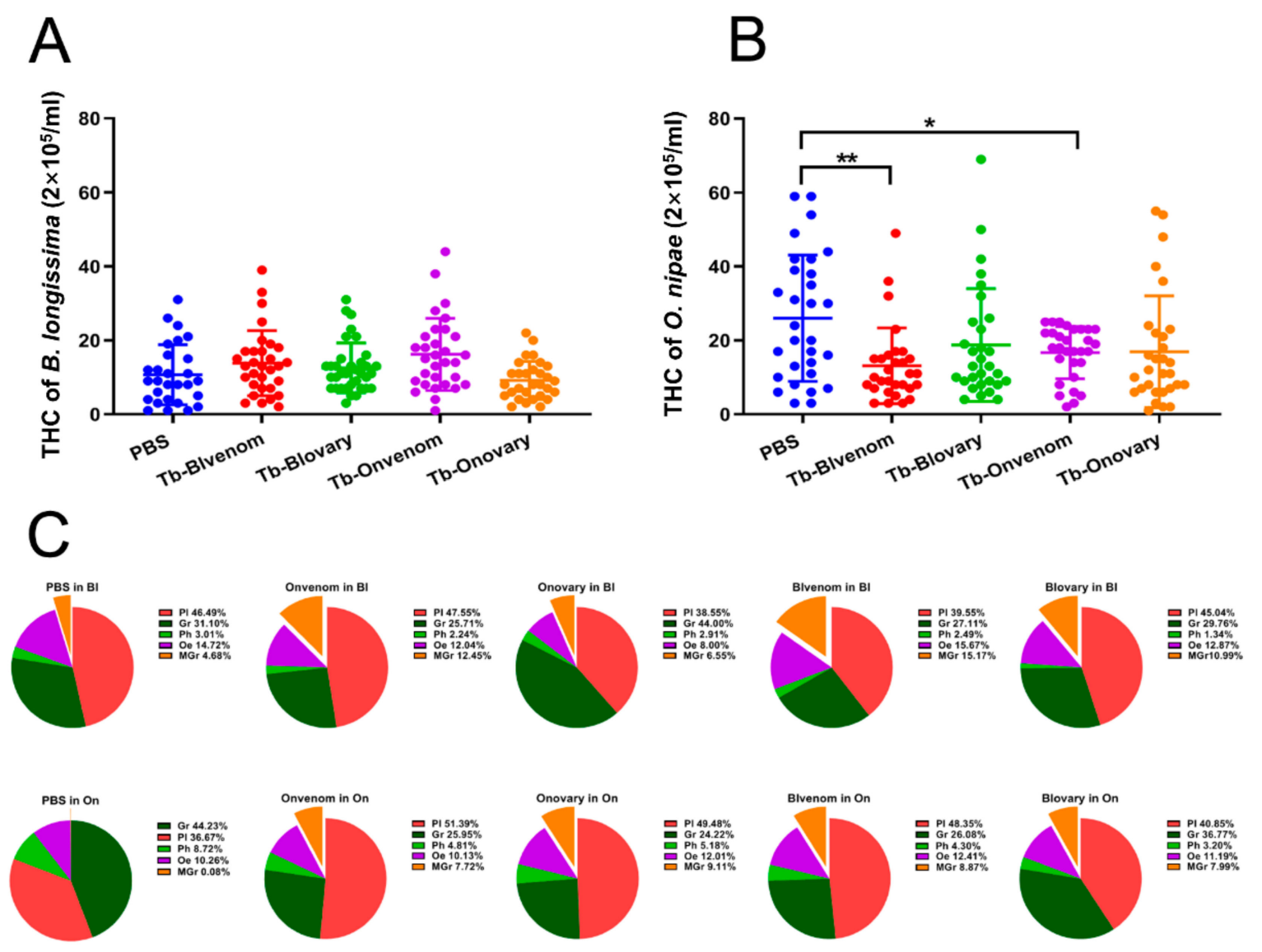

Figure 1. Comparison of cytotoxicity of venom or ovarian fluids injection against O. nipae and B. longissima pupae. (A,B) Total haemocyte counts (THCs) and (C) differential haemocyte counts (DHCs) of host pupae at $24 \mathrm{~h}$ after injection with venom or ovarian fluids. ${ }^{*}$ and ${ }^{* *}$ represent a significant difference at the $p<0.05$ level and $p<0.01$ level, respectively (Welch's/Brown-Forsythe ANOVA followed by Dunnett's T3 test).

\subsection{Virulence of Venom and Ovarian Fluids against Host Haemocyte Spreading}

Haemocyte spreading is recognised as a key indicator of cell viability. In this assay, rhodamine-phalloidin with red fluorescence was used to visualise the degrees of haemocyte extension after various treatments. The results indicate that in $O$. nipae pupae injected with PBS, haemocytes were highly organised and whipcord (Figure 2a,f). After the injection of venoms from the both wasp strains, plasmatocytes were extensively spread and extended pseudopods; the majority of the plasmatocytes were friable and had irregular polygonal morphologies (Figure 2b,d,g,i). Moreover, star-like F-actin clusters formed by the aggregates of actin cytoskeleton were observed all over the target plasmatocytes (Figure 2g,i). Notably, in the groups injected with the venom, the populations of granulocytes and macrogranulocytes were decreased (Figure $2 b, d, g, i$ ), and only a few completely intact macrogranulocytes and abundant free granules were observed compared to those in the groups injected with PBS or ovarian fluids (Figure $2 \mathrm{a}, \mathrm{c}, \mathrm{e}, \mathrm{f}, \mathrm{h}, \mathrm{j}$ ). Ovarian fluids had little effect on haemocytes (Figure 2c,e,h,j).

In B. longissima pupae, haemocytes in PBS treatment showed similar morphology to that in O. nipae pupae (Figure 2k,p). Ovarian fluids had little influences on haemocyte spreading (Figure $2 \mathrm{~m}, \mathrm{o}, \mathrm{r}, \mathrm{t}$ ). The effects of venom injection on haemocyte spreading were similar to that in $\mathrm{O}$. nipae pupae (Figure $2 \mathrm{~g}, \mathrm{l}, \mathrm{n}, \mathrm{s}$ ); $\mathrm{Tb}$-Onvenom had stronger effect on macrogranulocyte destruction than that observed in the case of Tb-Blvenom (Figure 2i,n, q,s). 


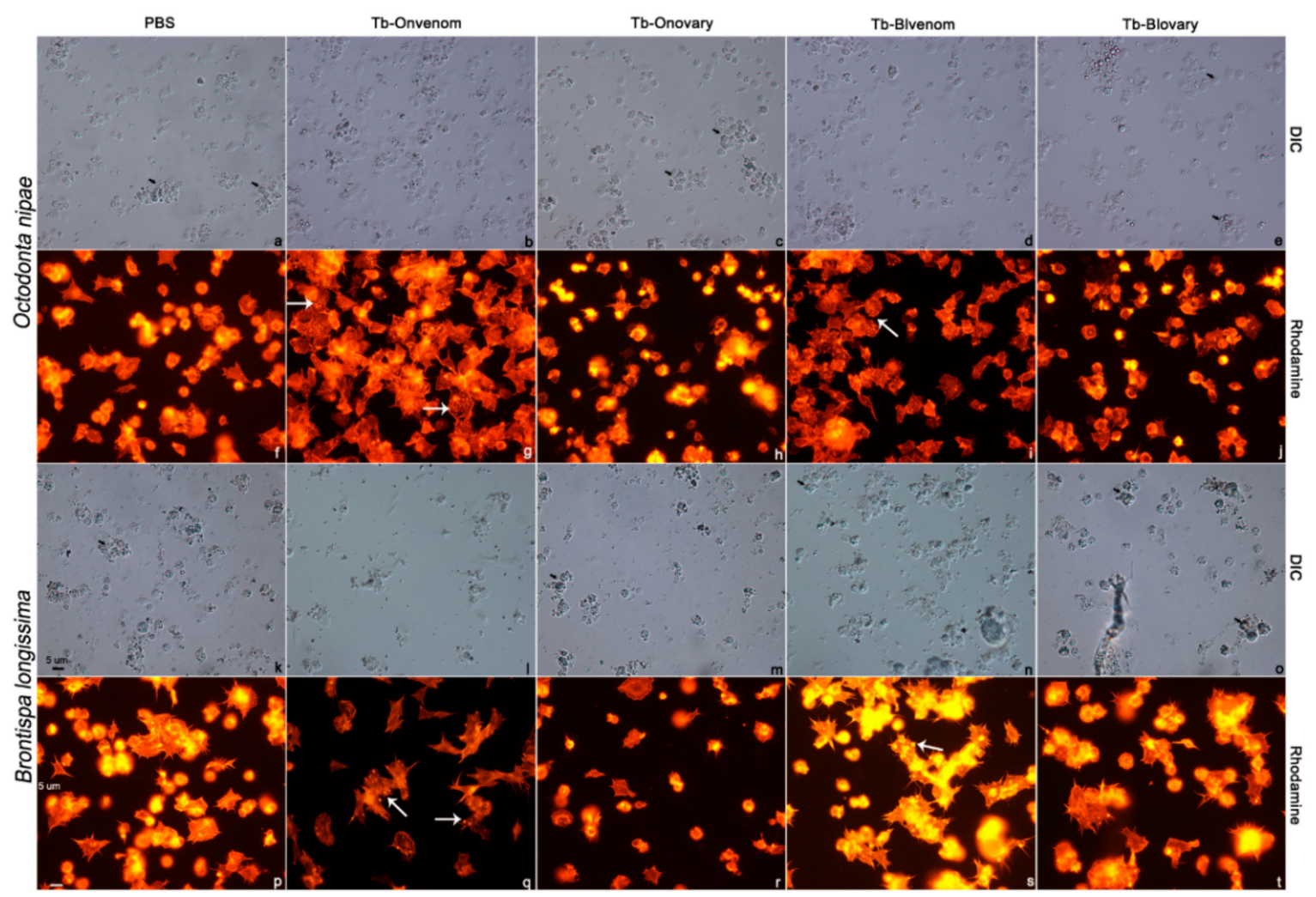

Figure 2. Haemocyte spreading assay in O. nipae and B. longissima pupae after the injection with virulent factors. (a-e,k-o) haemocytes visualised by differential interference microscopy; $(\mathbf{f}-\mathbf{j}, \mathbf{p}-\mathbf{t})$ fluorescence of rhodamine phalloidin-labelled cytoskeleton visualised by fluorescence microscopy. Star-like clusters in venom injection groups are marked by white arrows. Aggregates are marked by thick black arrows. Scale bars, $5 \mu \mathrm{m}$.

\subsection{Differential Virulence of Venom and Ovarian Fluids against Host Phagocytosis}

The virulence of venom or ovarian fluids against host phagocytosis was measured as the number of phagocytised FITC-labelled E. coli. In O. nipae pupae, the number of bacteria engulfed by haemocytes was significantly reduced after injection with venom or $\mathrm{Tb}$-Blovary, and the percentage of phagocytosis was approximately $48 \%$ of that observed in the PBS group (Figure 3A left panel; $\chi^{2}=99.553, \mathrm{df}=4, p<0.0001$ ). In B. longissima pupae, the venom from the both strains did not influence the phagocytosis; however, ovarian fluids from the both strains significantly inhibited the host phagocytosis by $48 \%$ (Figure $3 \mathrm{~A}$ right panel; $\chi^{2}=74.748, \mathrm{df}=4, p<0.0001$ ).

Notably, the percentage of macrogranulocytes plus granulocytes in B. longissima $(50.50 \%+12.28 \%)$ and $O$. nipae $(22.45 \%+45.54 \%)$ was increased after a challenge with E. coli compared to that after challenge with PBS (Figure 3B; B. longissima, $\chi^{2}=198.116$, $\mathrm{df}=3, p<0.0001 ;$ O. nipae, $\chi^{2}=86.652, \mathrm{df}=3, p<0.0001$. In the case of PBS, please see Figure 1C: B. longissima $(4.68 \%+31.10 \%)$, O. nipae $(0.08 \%+36.67 \%))$. In B. longissima, after the injection with venom or ovarian fluids, an $E$. coli challenge had little influence on the haemocyte composition (Figure 3B upper panel; $\chi^{2}=7.604, \mathrm{df}=12, p=0.107$ ). In $O$. nipae pupae after the injection with ovarian fluids, the percentage of macrogranulocytes in the E. coli-challenged pupae was continuously increasing; however, in the venominjected groups, higher number of plasmatocytes and lower number of macrogranulocytes were observed (Figure 3B down panel; $\chi^{2}=871.360, \mathrm{df}=12, p<0.0001$ ). Interestingly, venom induced individual haemocyte scattering, and ovarian fluids induced haemocyte aggregation in O. nipae; however, in B. longissima, the venom and ovarian fluids induced haemocyte aggregation (Figure $3 \mathrm{C}$ ). These results suggest that granulocytes are dominant in phagocytosis and aggregation (Figure 3C) and that macrogranulocytes are the main force of cellular immunity and are susceptible to the pathogen (Figure 3B). 

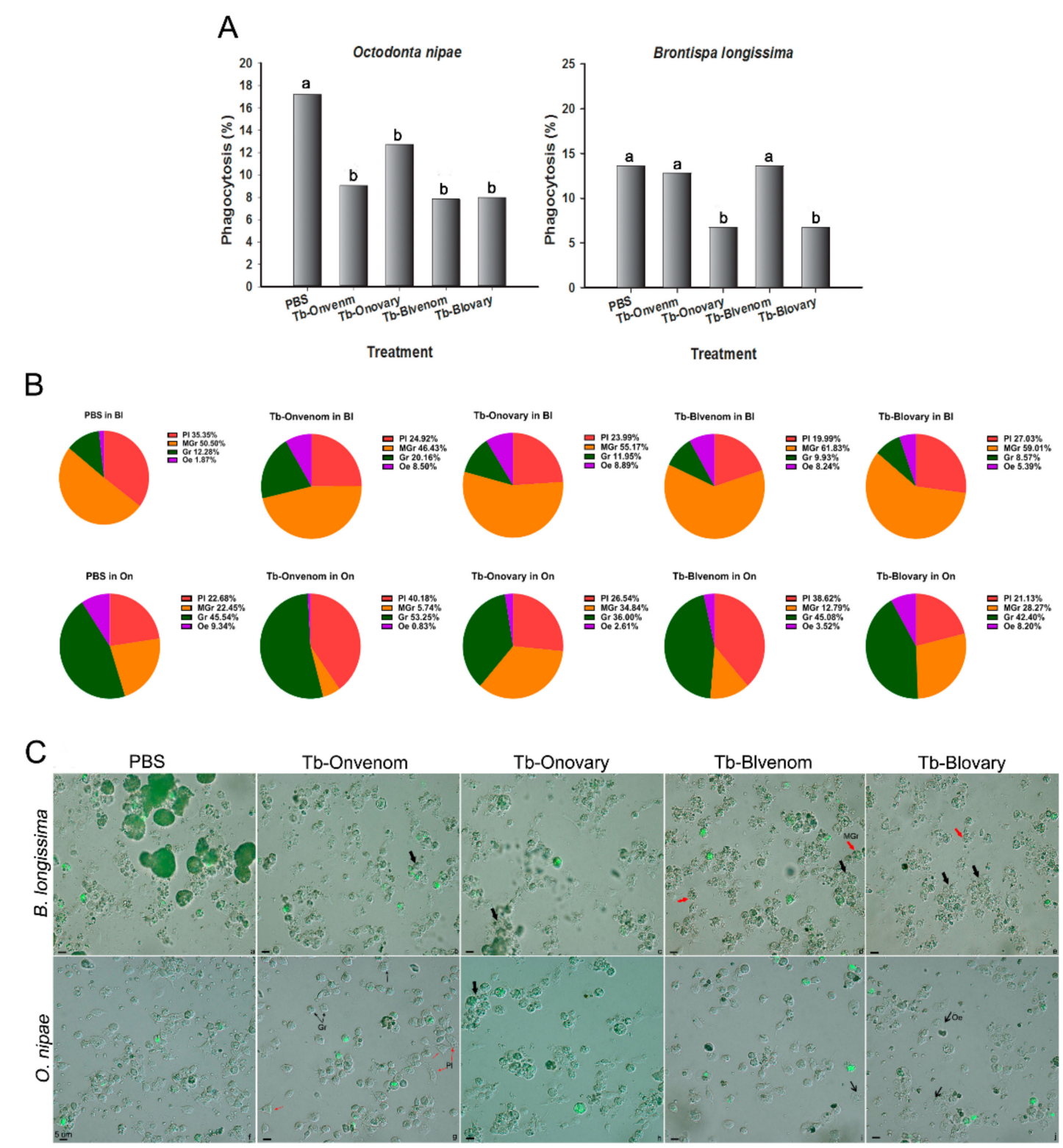

Figure 3. Haemocyte phagocytosis assay in O. nipae and B. longissima pupae after the injection with virulent factors. O. nipae or B. longissima pupae were pre-injected with virulent factors for $12 \mathrm{~h}$ followed by injections with heat-inactivated FITC-labelled E. coli for another $12 \mathrm{~h}$. Pupae injected with PBS were used as a control group. (A) Phagocytosis rate after treatments. Different lowercase letters represent a significant difference at the $p<0.001$ level $(\mathrm{R} \times \mathrm{C}$ chi-square test). (B) DHCs of O. nipae and B. longissima pupae injected with virulent factors and FITC-labelled E. coli. Pl, plasmatocyte (red); Gr, granulocyte (green); MGr, macrogranulocyte (orange); Oe, oenocytoid (purple). (C) Haemocytes visualised by differential interference microscopy and fluorescence images. Green fluorescence corresponds to FITC-labelled E. coli. Aggregates and macrogranulocytes are marked with thick black and red arrows, respectively. Pl and Gr are marked with thin red and black arrows, respectively. Scale bars, $5 \mu \mathrm{m}$.

\subsection{Differential Virulence of Venom and Ovarian Fluids against B. longissima and O. nipae Encapsulation}

Sephadex A-50 beads of equal diameter were selected to evaluate the encapsulation rate. In $O$. nipae pupae, the venom or ovarian fluids from both wasp strains induced an effective inhibition (approximately 55\%) of host haemocyte encapsulation (Figure 4A-C; $\left.F_{(4,20)}=18.665, p<0.001\right)$. In $B$. longissima pupae, ovarian fluids from both wasp strains induced a decrease in host encapsulation index (EI) by $50 \%$, while the venom did not influence the host encapsulation (Figure $4 \mathrm{~A}-\mathrm{C} ; F_{(4,20)}=14.733, p<0.001$ ). 
A

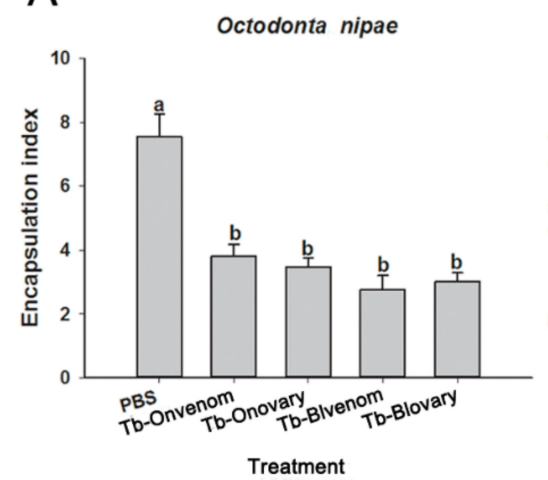

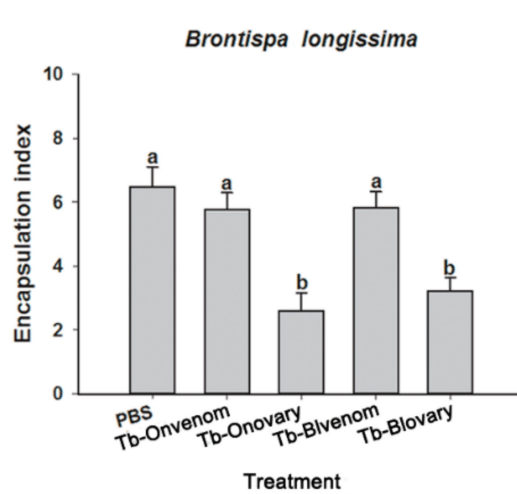

Treatment

Completely encapsulated beads

B Semi-encapsulated beads
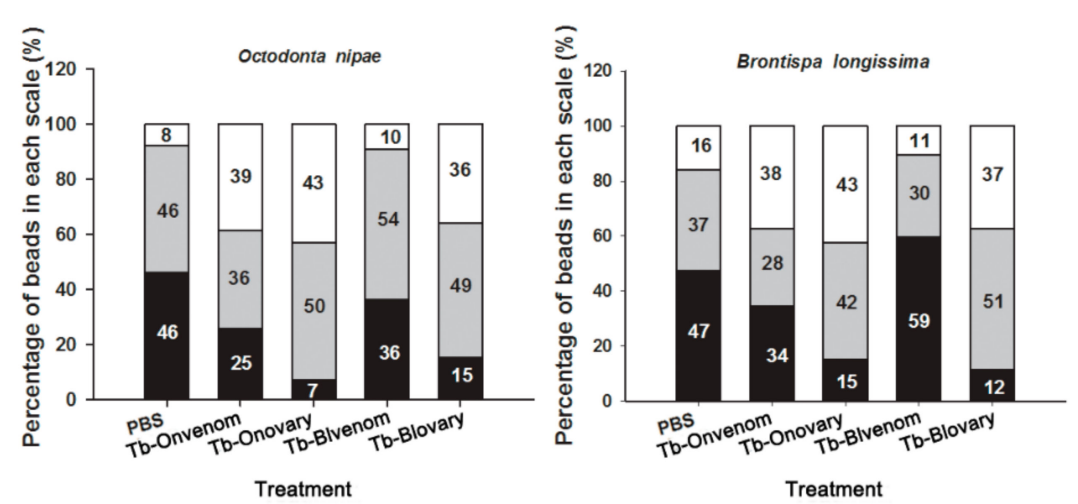

C

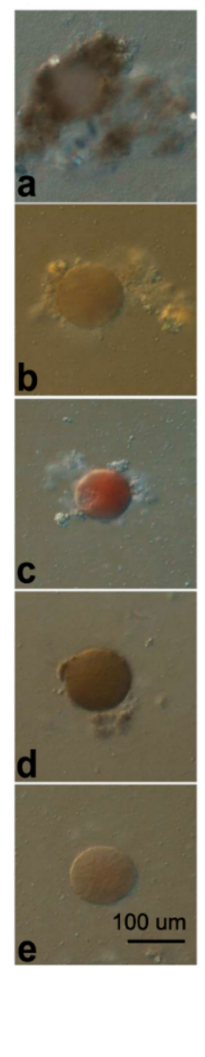

Figure 4. Effect of various virulent factors on haemocyte encapsulation. (A) Encapsulation index of $O$. nipae and B. longissima haemocytes at $24 \mathrm{~h}$ after treatments. (B) Number of beads of each scale in various treatments. The encapsulated beads were separated into three scales, depending on the number of haemocytes attached to the beads. (C) Beads with varying degrees of encapsulation. Different letters correspond to significant differences at the 0.05 level between different tissues (one-way ANOVA followed by Dunnett's test). Scale bars, $100 \mu \mathrm{m}$.

\subsection{Differential Virulence of Venom and Ovarian Fluids against Host PO Activity}

The activation of pro-PO (PPO) is an important part of humoural immunity. Interestingly, the same volume of haemolymph had substantially higher $\mathrm{PO}$ activity in $B$. longissima than that in O. nipae (Figure S2; $t=7.880, \mathrm{df}=13, p<0.0001$ ). After the injection of the venom or ovarian fluids into $B$. longissima or $O$. nipae pupae, the haemolymph spontaneously became black within $100 \mathrm{~min}$ (Figure $5 \mathrm{~A}$ ), and the $\mathrm{PO}$ activity of the haemolymph was increased by 2-3 times (Figure $5 \mathrm{~B}, \mathrm{C} ; \mathrm{On}, F_{(2,10)}=16.74, p<0.001 ; \mathrm{Bl}, F_{(2,11)}=52.69$, $p<0.0001)$; however, the colour of the haemolymph in the PBS group was only slightly changed within 100 min (Figure 5A). Usually, PO activity in the haemolymph can be induced by microbial elicitors, such as E. coli. However, in O. nipae pupae injected with $\mathrm{Tb}$-Onvenom or Tb-Onovary, the addition of $E$. coli to the haemolymph did not induce an increase in PO activity (Figure 5D). Similarly, the addition of E. coli did not induce an increase in $\mathrm{PO}$ activity in $\mathrm{B}$. longissima pupae injected with $\mathrm{Tb}$-Onvenom. However, in $\mathrm{Tb}$ Blvenom- or Tb-Blovary-injected host pupae (in B. longissima and O. nipae), the PO activity was still induced by E. coli (Figure 5D,E; On, $F_{(4,16)}=8.592, p<0.001 ; \mathrm{Bl}, F_{(4,20)}=7.984$, $p<0.001)$. 
A

\section{O. nipae}

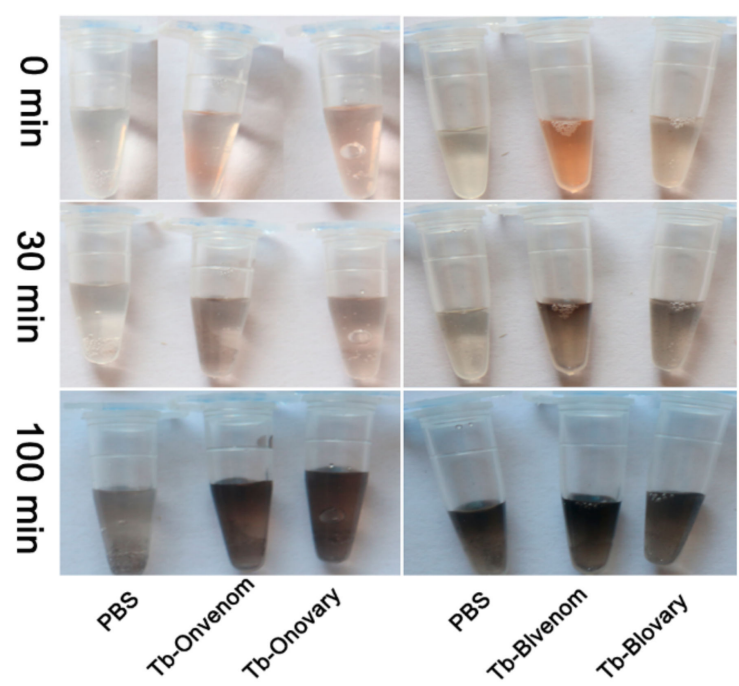

B

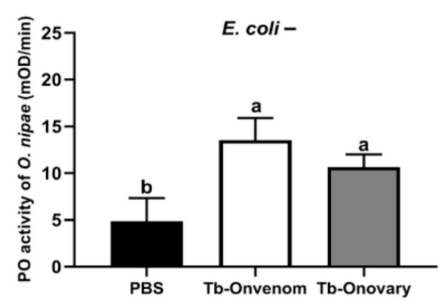

C

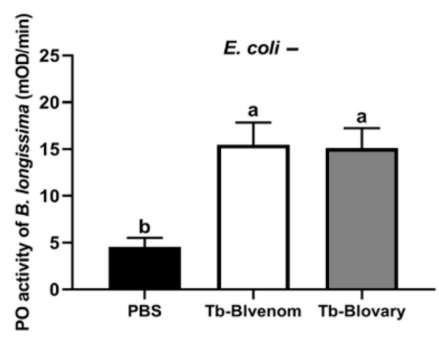

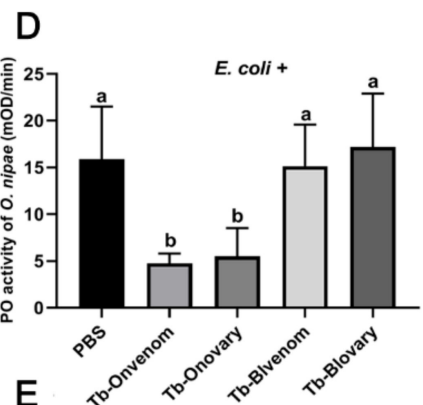

E

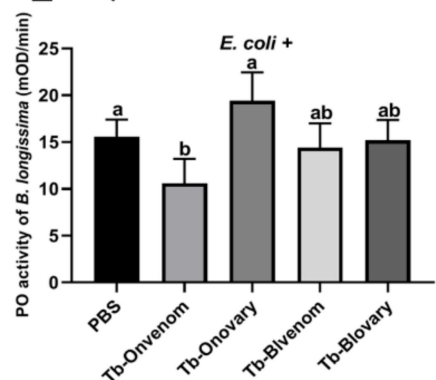

Figure 5. Effect of virulent factors on phenoloxidase (PO) activity $24 \mathrm{~h}$ post injection. (A) Melanisation of $O$. nipae and B. longissima haemolymph after the injection with venom or ovarian fluids. (B,C) PO activity of O. nipae or B. longissima haemolymph after venom and ovarian fluids injection. (D,E) PO activity of haemolymph collected from $O$. nipae or B. longissima pupae after the injection with various virulent factors and incubated with heat-inactivated E. coli for $10 \mathrm{~min}$. Average $V_{\max }$ was used to evaluate PO activity. Error bars indicate standard deviations of the mean of five independent biological replications. Different letters correspond to significant differences at the 0.05 level between different tissues (one-way ANOVA followed by Dunnett's test).

\subsection{Venom Promotes B. longissima and O. nipae Fat Body Lysis}

Degradation of the host fat body was determined by ORO and H\&E staining. In O. nipae, the fat body began to lyse $24 \mathrm{~h}$ after the injection with venom (Figure S3g,i), and was severely degraded and decomposed at $48 \mathrm{~h}$ (Figure $6 \mathrm{~b}, \mathrm{~d}, \mathrm{~g}, \mathrm{i})$. Tb-Blvenom caused a stronger effect on fat body lysis compared to that observed in the case of Tb-Onvenom (Figure $6 \mathrm{~b}, \mathrm{~d}, \mathrm{~g}, \mathrm{i})$. In detail, venom induced fat body decomposition into small pieces, resulting in appearance of abundant lipid droplets (Figure $6 \mathrm{~b}, \mathrm{~d}$ ) and severe degradation of adipose cell (Figure $6 \mathrm{~g}, \mathrm{i})$. After injection with PBS or ovarian fluids, a high number of large fragments of fat body remained (black arrow, Figure $6 \mathrm{a}, \mathrm{c}, \mathrm{e}$ ), and adipose cells were intact and tightly arranged (Figure $6 \mathrm{f}, \mathrm{h}, \mathrm{j}$ ). In B. longissima, $24 \mathrm{~h}$ after the injection with the venom, the fat body was severely decomposed and degraded, and a more pronounced effect was caused by Tb-Blvenom (Figure $6 \mathrm{~b}, \mathrm{~d}, \mathrm{~g}, \mathrm{i}$ ). Notably, the fat body in B. longissima pupae appeared to be easily degraded even in the PBS or ovarian fluid-injected groups, thus making it difficult to evaluate suitable sections (Figure 6 and Figure S4). 

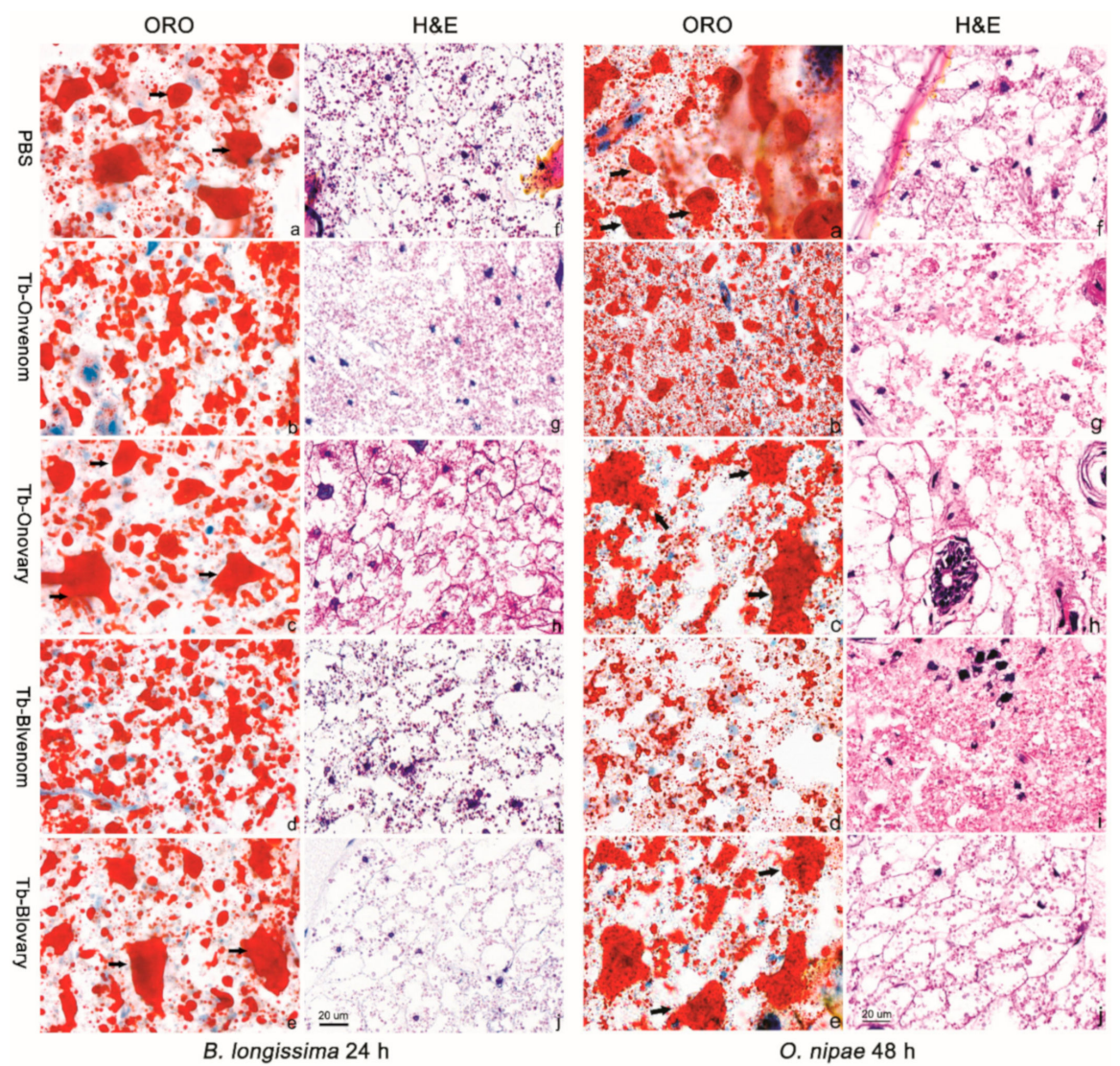

Figure 6. Effect of virulent factors on B. longissima and O. nipae pupae fat body lysis at $24 \mathrm{~h}$ and $48 \mathrm{~h}$ post injection, respectively. ORO and $\mathrm{H} \& \mathrm{E}$ stained fat body tissues of $B$. longissima and O. nipae pupae after the injection with PBS (a,f), Tb-Onvenom $(\mathbf{b}, \mathbf{g})$, Tb-Onovary $(\mathbf{c}, \mathbf{h})$, Tb-Blvenom $(\mathbf{d}, \mathbf{i})$ or Tb-Blovary $(\mathbf{e}, \mathbf{j})$; original magnification, $40 \times$. Larger pieces of fat body are marked by thick black arrows in the ORO sections. Scale bar, $20 \mu \mathrm{m}$.

\subsection{Comparison of Transcriptome Analysis between O. nipae and B. longissima}

Comparative transcriptome analysis was used to visualise the differentiation of physiological dimension between $O$. nipae and B. longissima. A pair of genes with a $70 \%$ coverage and an E-value cut-off of $10^{-5}$ was considered to be a homologous gene between the two hosts. The homologous comparison results indicate that only 6443 genes were conserved, and $83.18 \%$ and $73.31 \%$ unigenes were unique for O. nipae and B. longissima, respectively (Figure 7A, Figure S5 and Table S1).

The transcriptome profiles of $B$. longissima and $O$. nipae pupae were significantly influenced $24 \mathrm{~h}$ after the infection with $T$. brontispae. Using the criteria of $\log _{2}$-fold $>1.5$ and FDR $<0.05,706$ unigenes (577 upregulated and 129 downregulated) were significantly differentially expressed in O. nipae (Figure S6); in B. longissima, the majority of the differentially expressed genes were upregulated (242 upregulated and 1 downregulated, Figure S6). For an additional focus on the conserved genes related to lipid metabolism, immune system and cell motility, we performed a comparative expression profile for these genes in samples prepared after the infection by T. brontispae. The results indicate that the majority of the genes in O. nipae are downregulated or unchanged after parasitism, while in B. longissima, the genes were upregulated (Figure 7B-D). 
A

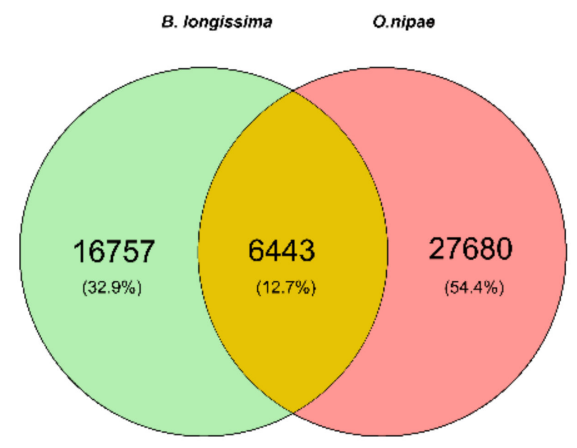

C

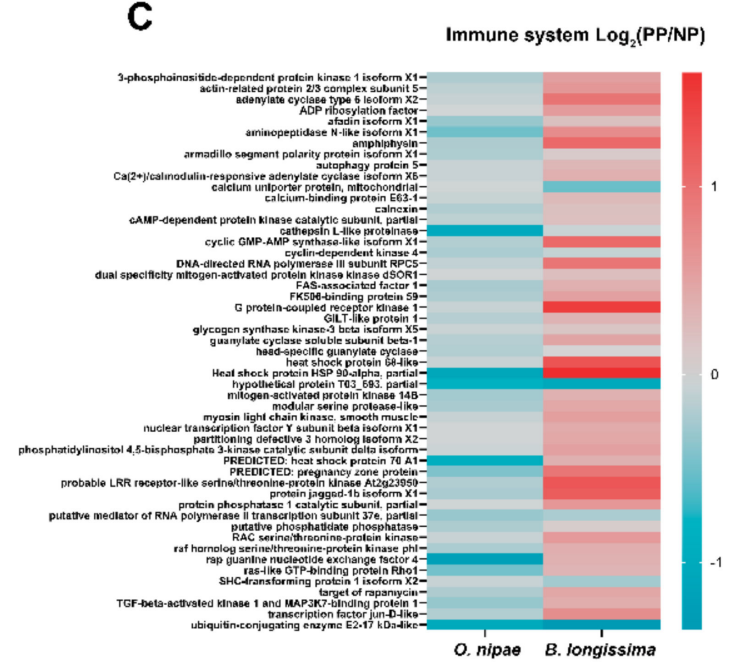

B

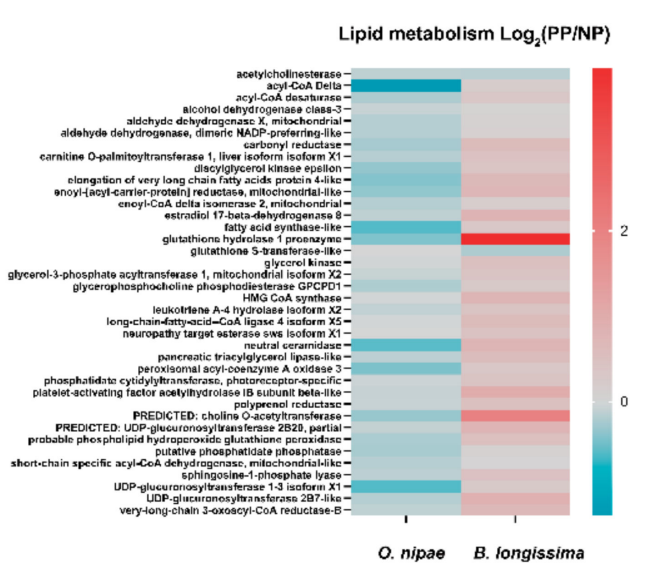

D

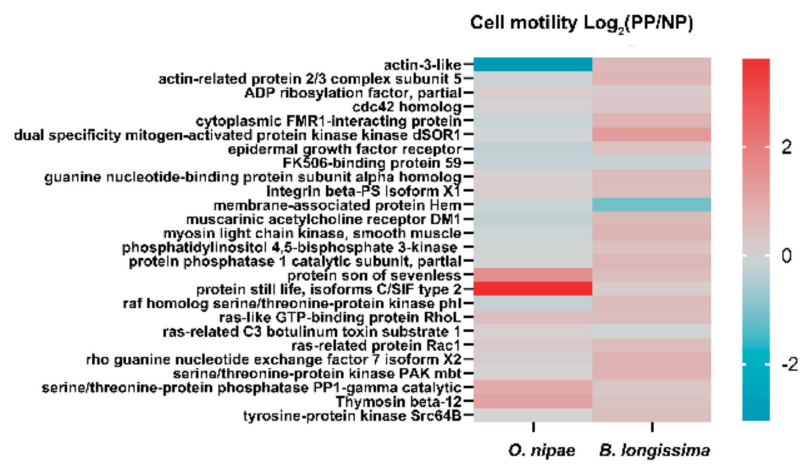

Figure 7. Comparison of transcriptomic analysis of B. longissima and O. nipae. (A) Homology analysis of $O$. nipae and B. longissima unigenes with a $70 \%$ coverage and an E-value cut-off of $10^{-5}$; (B-D) Differential expression analysis of lipid metabolism-related (B), immune system-related (C), and cell motility-related (D) homology genes in $O$. nipae and B. longissima between non-parasitised (NP) and parasitised pupae (PP).

\section{Discussion}

Venom genes encode for multiple factors to target the humoural and cellular immune systems and nutritional metabolism of the host to help parasitoid offspring avoid the killing activity of host defence and gain enough energy supply [52-55]. In the present study, venom induced cytotoxicity in haemocytes, including a decline in THCs, a reduction in granulocyte percentage and an increase in percentage of plasmatocytes and macrogranulocytes in O. nipae pupae. Similarly, in the Pimpla turionellae-Galleria mellonella system, venom injection induced a reduction in the number of granulocytes and an increase in the number of plasmatocytes; more importantly, such injection induced the formation of vacuoles within the cytoplasm in granulocytes with eventual death of granulocytes $[29,56]$. Macrogranulocytes are activated granulocytes, which are characterised by large cytoplasmic vacuoles, undergo subsequent apoptosis and eventually discharge a large amount of materials [57-59]. In our system, an increase in percentage of macrogranulocytes was detected, and we thus hypothesise that the T. brontispae venom induces the unloading of macrogranulocytes (weapons-granulocytes), that the transformation of granulocytes into macrogranulocytes without a supplement results in a decrease in granulocytes, and subsequently, that macrogranulocytes undergo apoptosis leading to a decrease in THCs.

Additionally, venom can significantly inhibit the spreading of plasmatocytes for a number of reasons. The inhibition of F-actin development within haemocytes is universally associated with disturbance of actin cytoskeleton [60]. Cytoskeleton rearrangements and microfilaments are involved in encapsulation and phagocytosis [61,62]; hence, the forma- 
tion of star-like actin clusters (the actin aggregates) in plasmatocytes in the venom injection group in the present study most likely caused the inhibition of haemocyte spreading, encapsulation and phagocytosis. Similarly, the concerted action of two toxins, TccC3 and TccC5 adenosine diphosphate-ribosyltransferases from Photorhabdus luminescens, induced the clustering of F-actin in Galleria mellonella haemocytes, thus inhibiting phagocytosis of the target haemocytes [63].

PPO is synthesised in the haemocytes and is released into the haemolymph due to the rupture of haemocytes [64,65]. PPO is usually synthesised in oenocytoids [66]. Recently, it has been demonstrated that PPO can also be produced in plasmatocytes (lamellocytes) and granulocytes. In Drosophila, PPO3 produced by lamellocytes emerged as a novel defence mechanism against parasitoid wasps [67]. PO activity was determined in granulocytes in Mythimna separata [68]. A previous study demonstrated that in O. nipae, in addition to oenocytoids, granulocytes were PPO/PO-positive haemocytes [69]. Granulocytes from O. nipae pupae treated with Triton $\mathrm{X}-100$ can be stained with an anti-PPO antibody to produce a strong fluorescence signal (Zhang et al., unpublished data). Thus, we hypothesise that in O. nipae, granulocytes can also produce PPO. After the injection of venoms or ovarian fluids, the percentage of oenocytoids did not change; hence, an increase in the $\mathrm{PO}$ activity may be attributed to the apoptosis of macrogranulocytes rather than to the rupture of oenocytoids. Moreover, the apoptosis of macrogranulocytes and the subsequent release of their inclusions is related to fat body decomposition and degradation in Helicoverpa armigera [59]. This mechanism may be involved in induction of the lysis of host fat body by venom of T. brontispae.

Antagonistic interactions between parasitoids and their hosts drive evolution and generate diversity leading to coevolution of both species [8]. Trade-offs caused by strength of selection in the host-parasitoid systems are characterised by the host resistance strategies and parasitoid virulence tactics [9]. In our system, evolutionary events occur only in the parasitoid. Venoms from $\mathrm{Tb}$-On and $\mathrm{Tb}$-Bl displayed virulent activities towards the two hosts, and Tb-Onvenom was more virulent towards plasmatocyte spreading, granulocyte function and $\mathrm{PO}$ activity than Tb-Blvenom, suggesting a variability in the virulence of T. brontispae venom under $O$. nipae selection. In the case of ovarian fluids with complex function, some of these functions may be complementary to the effects of venom [70], others may act as an antidote to reverse the effects of the venom [71], and some may function independently [72]. Tb-Blovary was able to suppress encapsulation and phagocytosis of E. coli in both hosts; however, Tb-Onovary inhibition targeted only B. longissima Thus, both venom and ovarian fluids contribute to the inhibition of host immunity by targeting $B$. longissima pupae hosts; however, the contribution of venom to the inhibition of $O$. nipe immunity is higher than the impact of ovarian fluid. Regardless of the host, venoms from both strains were virulent in host fat body lysis. Overall, these results indicate that the evolution of venoms and ovarian fluids in T. brontispae occurs in response to host selection.

Venom compositions in parasitoid wasps, which are important counter-resistance traits, are undergoing rapid evolution while facing various hosts, suggesting a potential for adaptation to new hosts [45]. In the case of L. boulardi, variation in the venom counterresistance in facing $D$. yakuba is correlated to variability of the effects on the PO cascade and plasmatocyte number; in D. melanogaster, this phenomenon is related to the effects on the lamellocyte number and morphology [38,73,74]. Multiple inheritance systems transfer accommodation and channel stress-induced variability between the generations drive longterm persistence and stress-induced evolutionary adaptations [75]. It seems reasonable that T. brontispae generates evolutionary adaptations in B. longissima and O. nipae. Substantial differences in physiological dimension between $B$. longissima and $O$. nipae indicated by comparative transcriptome analysis induce different types of stress, such as immune defence, energy supply and development time and space, in T. brontispae. This is the reason why $T$. brontispae has to adjust when targeting O. nipae. The stronger disruptive effect of $\mathrm{Tb}$-Onvenom on plasmatocyte spreading, granulocyte function and $\mathrm{PO}$ activity may be due to the stronger selection stress of cellular immunity from O. nipae. 
Local adaptation can be a major cause of evolution and speciation [30,76]. In T. brontispaeB. longissima/O. nipae system, $\mathrm{Tb}-\mathrm{On}$ or $\mathrm{Tb}-\mathrm{Bl}$ did not demonstrate differences in parasitism rate, emergence rate and sex ratio towards O. nipae and B. longissima (Lin et al., unpublished data). These indicate that $\mathrm{Tb}-\mathrm{On}$ or $\mathrm{Tb}-\mathrm{Bl}$ could also perform well when encountering non-local host (the non-local host of Tb-On is B. longissima, and the non-local host of $\mathrm{Tb}-\mathrm{Bl}$ is $\mathrm{O}$. nipae). Thus, this wasp has ample evolutionary plasticity, and the theory of local adaptation does not apply to this system because we have shown that the virulence of the venom is enhanced under the pressure of O. nipae-driven selection. Similarly, in the Cotesia melitaearum and Melitaea cinxia system, no local adaptation was found in different populations; however, there was a local genetic variation on a short-term scale for hundreds of generations [77]. In the Lysiphlebus fabarum and Aphis fabae system, variations in the virulence of parasitoids within four different populations was observed; however, the lack of genetic differentiation or genotype-specific interactions suggested a lack of local adaptation, regardless of genetic variation [78]. The comparison of susceptibility of Drosophila melanogaster and virulence of Asobara tabida indicates that local adaptation was not detected across European populations [74]. Thus, local adaptation may exist in the system in which the parasitoid performed various adjustments to host populations, similar to the Drosophila and L. boulardi system [79] and the Galerucella and A. parviclava system [9].

\section{Materials and Methods}

\subsection{Hosts and Wasp Strains}

Brontispa longissima and O. nipae were reared under the conditions of $75 \pm 5 \%$ relative humidity, $25 \pm 1{ }^{\circ} \mathrm{C}$ and a photoperiod of 12:12 (light: dark), as described previously [80], and they were supplied with fresh leaves of Canary Island date palm, Phoenix canariensis Hort. Ex Chabaud, as a diet. The origins of T. brontispae and two strains of the wasps were as described previously [50,51]. Briefly, T. brontispae- $\mathrm{Bl}$ (Tb-Bl) was cultivated with one-day-old (newly exuviated) B. longissima pupae, and T. brontispae-On (Tb-On), a derived sub-population of $\mathrm{Tb}-\mathrm{Bl}$, underwent a host transposition from B. longissima to O. nipae consecutively for six years (120 generations). Wasp strains were maintained under the same conditions as the hosts. After emergence, adult wasps were fed on a streak of $10 \%$ sucrose solution in plastic containers as described previously [50]. Because female wasps have the highest rate of parasitism on the newly exuviated host pupae, one-day-old host pupae were selected for all subsequent experiments unless specified otherwise.

\subsection{Preparation of Venom and Ovarian Fluids from the Two Stains}

Female adult wasps without parasitism experience of each strain were selected for collection of the venom and ovarian fluids $24-48 \mathrm{~h}$ after emergence. The female wasps were dissected on ice to separate the venom apparatus and ovary, as described previously [21]. Approximately 200 venom apparatuses or ovaries from each strain were combined in $40 \mu \mathrm{L}$ PBS $\left(0.01 \mathrm{M}, 8 \mathrm{~g} / \mathrm{L} \mathrm{NaCl}, 0.20 \mathrm{~g} / \mathrm{L} \mathrm{KCl}, 1.44 \mathrm{~g} / \mathrm{L} \mathrm{Na}_{2} \mathrm{HPO}_{4}\right.$ and $0.24 \mathrm{~g} / \mathrm{L} \mathrm{KH}_{2} \mathrm{PO}_{4}, \mathrm{pH}$ 7.4 ) in a PCR tube (5 equivalents per $\mu \mathrm{L}$ ). Then, the venom apparatuses from $\mathrm{Tb}-\mathrm{On}$ and $\mathrm{Tb}-\mathrm{Bl}$ were homogenised by a tissue grinder and centrifuged at 12,000 $\mathrm{g}$ for $10 \mathrm{~min}$ at $4{ }^{\circ} \mathrm{C}$ to collect the supernatant, after which the samples were labelled as Tb-Onvenom and $\mathrm{Tb}$-Blvenom, respectively; ovaries from $\mathrm{Tb}-\mathrm{On}$ and $\mathrm{Tb}-\mathrm{Bl}$ were dissected and centrifuged at $10,000 \mathrm{~g}$ for $10 \mathrm{~min}$ at $4{ }^{\circ} \mathrm{C}$ to remove eggs and cellular debris, and the resulting supernatants were labelled as $\mathrm{Tb}$-Onovary and $\mathrm{Tb}$-Blovary, respectively. All prepared samples were divided into small aliquots in a PCR tube and stored at $-80^{\circ} \mathrm{C}$ until use.

\subsection{Assay of Total Haemocyte Counts (THCs) and Differential Haemocyte Counts (DHCs)}

To test the virulence of $\mathrm{T}$. brontispae of the $\mathrm{Tb}-\mathrm{Bl}$ and $\mathrm{Tb}$-On strains on its respective host haemocytes, $207 \mathrm{nl}$ of Tb-Onvenom or Tb-Onovary (about one equivalent to each host) was injected into O. nipae pupae, and the same volume of Tb-Blvenom or Tb-Blovary was injected into B. longissima pupae by a NANOLITER 2010 (WPI, Sarasota, FL, USA). PBS injection was set as a control group for the corresponding host. One microliter of 
host haemolymph was harvested from each treatment at various time points $(12,24$, 48,72 and $96 \mathrm{~h}$ ) after the injection. The haemolymph (one microliter) was then diluted with four microliters of Schneider's Drosophila medium (Thermo Fisher, Waltham, MA, USA) and subsequently loaded on a haemocytometer (Shanghai Qiujing Corporation, Shanghai, China) for THC and DHC analyses; the samples were imaged and analysed by fluorescence and differential interference contrast microscopy with the NIS-N-viewer software (Nikon Ni-U, Tokyo, Japan). Each treatment at each time point was performed in at least 30 replicates.

To test whether the changes in the quality and/or quantity in the venoms and ovarian fluids of the two strains are responsible for the variations in parasitoid virulence, $\mathrm{Tb}$ Onvenom or Tb-Onovary was injected into B. longissima pupae and Tb-Blvenom or $\mathrm{Tb}$ Blovary was reciprocally injected into $O$. nipae pupae. Because the virulence of the wasp had the visible effects (e.g., THCs and DHCs) on the host $24 \mathrm{~h}$ after the injection of the venom or ovarian fluids, host pupae were collected $24 \mathrm{~h}$ after the injection in all subsequent experiments unless specified otherwise.

\subsection{In Vitro Haemocyte Spreading Assay}

In this assay, $3 \mu \mathrm{L}$ of the haemolymph drawn from five pupae of $O$. nipae at $24 \mathrm{~h}$ post-injection with $207 \mathrm{nl}$ of Tb-Onvenom, Tb-Onovary, Tb-Blvenom, Tb-Blovary or PBS was mixed with $2 \mu \mathrm{L}$ physiological saline solution (PS) in each treatment; in the case of $B$. longissimi, a $5-\mu \mathrm{L}$ haemolymph sample from five injected pupae was not diluted with PS. The collected haemolymph was incubated at room temperature for $30 \mathrm{~min}$ on a lysine-coated glass (CITOGLAS ${ }^{\circledR}$ SHIATI ${ }^{\circledR}$, China) in a wet black box and the glass was washed. The attached haemocytes were fixed by $4 \%$ paraformaldehyde (diluted with PBS) for $15 \mathrm{~min}$ and washed with PBS. The haemocytes were then treated with $0.1 \%$ Triton X-100 (diluted with PBS) for $5 \mathrm{~min}$, washed and incubated with $20 \mu \mathrm{L} \mathrm{1 \%} \mathrm{BSA} \mathrm{for} 1 \mathrm{~h}$ in a wet black box. Then, $10 \mu \mathrm{L} 10 \%$ rhodamine phalloidin (diluted in PBS) (Thermo Fisher) was added to target F-actin for $30 \mathrm{~min}$, and the samples were washed. Subsequently, $10 \mu \mathrm{L}$ $1 \mu \mathrm{g} / \mu \mathrm{L}$ DAPI (4',6-diamidino-2-phenylindole $4^{\prime}$,6-diamidino-2-phenylindole) was added to stain the nuclei for $10 \mathrm{~min}$. The treated haemocytes were observed in the blue and red channels to detect fluorescence of the nuclei and cytoskeleton, respectively, by fluorescence and differential interference contrast microscopy. Each treatment included four biological replicates, and at least 2000 haemocytes were counted.

\subsection{In Vivo Phagocytosis Assay}

Approximately $138 \mathrm{nl}$ of heat-inactivated Fluorescein isothiocyanate (FITC)-labelled E. coli $\left(\mathrm{OD}_{600}=0.5\right)$ was injected into each host pupa (B. longissima or O. nipae) that was pre-injected with $207 \mathrm{nl}$ of Tb-Onvenom, Tb-Onovary, Tb-Blvenom, Tb-Blovary or PBS for $12 \mathrm{~h}$ (a total of ten treatments), and the treated pupae were incubated for another $12 \mathrm{~h}$. For $O$. nipae pupae, $3 \mu \mathrm{L}$ of the haemolymph collected from five pupae was mixed with $2 \mu \mathrm{L}$ PS; for B. longissima, $5 \mu \mathrm{L}$ of the haemolymph collected from five pupae was not diluted with PS. Then, the haemolymph was dropped onto a lysine-coated glass and incubated for $30 \mathrm{~min}$ at room temperature in a wet black box. After the incubation, the haemocytes were washed three times with PBS and then observed under a fluorescence and differential interference contrast microscope. The images and the phagocytosis rate were analysed with the NIS-N-viewer software. Each treatment included four biological replicates, and at least 2000 haemocytes were counted.

\subsection{In Vitro Haemocyte Encapsulation Assay}

Approximately $207 \mathrm{nl}$ of Tb-Onvenom or Tb-Onovary was injected into O. nipae and $B$. longissima pupae; similarly, the same volume of Tb-Blvenom or Tb-Blovary was injected into O. nipae and B. longissima pupae. PBS injections into O. nipae and B. longissima pupae were used as the control groups. At $24 \mathrm{~h}$ post-injection, five microliters of the haemolymph was harvested from at least five pupae in each treatment and mixed with five microliters 
of Schneider's Drosophila medium that contained ten dextran gel beads (DEAE-Sephadex A-50; beads of equal diameter were selected for Congo red staining). The mixture was incubated at room temperature for $24 \mathrm{~h}$ on a tube revolver (TR-02U, Crystal Industry, Dallas, TX, USA) and rotated at a speed of $10 \mathrm{rpm}$; then, the encapsulated or unencapsulated beads were observed under a Nikon differential interference contrast microscope and imaged with the NIS-Elements D 4.30.00 software. The encapsulation index (EI) was quantified as the thickness $(\mathrm{T})$ and area $(\mathrm{A})$ of haemocytes attached to the beads according to the previously described method [81]. Each treatment was performed in at least five replicates.

\subsection{In Vitro Haemolymph Phenoloxidase (PO) Activity Assay}

Approximately $2.5 \mu \mathrm{L}$ of the haemolymph from five $B$. longissima or $O$. nipae pupae at $24 \mathrm{~h}$ post-injection with $207 \mathrm{nl}$ Tb-Onvenom, Tb-Onovary, Tb-Blvenom, Tb-Blovary or PBS was mixed with $7.5 \mu \mathrm{L}$ Schneider's Drosophila medium containing heat-inactivated E. coli as stimulus and incubated at room temperature for $10 \mathrm{~min}$; then, PBS was added to a final volume of $70 \mu \mathrm{L}$. The PO activity was assayed as described previously [82,83]. In brief, for each sample, $20 \mu \mathrm{L}$ pretreated haemolymph was mixed with $30 \mu \mathrm{L}$ PBS in a 96 well microtiter plate (JingAn Biological, Shanghai, China); then, $100 \mu \mathrm{L}$ L-3,4-dihydroxyphenylalanine (L-DOPA, $8 \mathrm{mg} / \mathrm{mL}$, dissolved in PBS) was added to each well to a final volume of $150 \mu \mathrm{L}$. PO activity was measured by a spectrophotometer (SpectraMax, Molecular Devices Corporation, USA) with the following settings: absorbance, $490 \mathrm{~nm}$; intervals time, $60 \mathrm{~s}$; total reaction time, $30 \mathrm{~min}$; temperature, $28{ }^{\circ} \mathrm{C}$. Three technical replicates were used for each assay to obtain an average $V_{\max }$ (the slope value of the reaction curve) to assay the PO activity. For each treatment, PO activity was averaged from five biological replicates.

\subsection{Virulence of the Venom and Ovarian Fluids on Host Fat Body Lysis}

Haematoxylin-eosin (H\&E) and Oil Red O (ORO) staining were used to measure the virulence of the venom and ovarian fluids on host fat body lysis. The B. longissima or O. nipae pupae at $24 \mathrm{~h}$ post-injection with $207 \mathrm{nl}$ Tb-Onvenom, Tb-Onovary, Tb-Blvenom, $\mathrm{Tb}$-Blovary or PBS were pierced at the appendage with a small insect needle and infiltrated in $4 \%$ paraformaldehyde for $2 \mathrm{~h}$. The treated pupae were shipped to Wuhan Servicebio Technology Co., Ltd., Wuhan, China, for microscopic observation. Each pupa was fixed with SAKURA Tissue-Tek ${ }^{\circledR}$ O.C.T. compound (SAKURA, Tokyo, Japan) and sectioned at a thickness of $10 \mu \mathrm{m}$ (Cryostar NX70, Thermo Scientific, Waltham, MA, USA). The ultrathin sections were mounted on glass slides and subjected to ORO staining for evaluation of dispersion of lipid droplets [84,85]. For H\&E staining, 4\% paraformaldehyde-fixed pupae were dehydrated in a gradient of alcohol, embedded in paraffin (Taikang, TKY-BMB, Wuhan, Hubei, China) and sectioned at $4 \mu \mathrm{m}$ of thickness by a freezing microtome (SLEE, MNT, Mainz, Germany). Paraffin-embedded pupae sections were dewaxed, rehydrated, and stained with H\&E (C0105, Solarbio Science \& Technology, Beijing, China). Then, xylene was used to dehydrate and clear the sections, and the coverslips were mounted with synthetic media (Permount, Zhanyun, Shanghai, China). The sections were analysed and the images were recorded using a biological microscope (Olympus, CX43, Shinjuku-ku, Tokyo, Japan); images were processed by the Caseviewer software.

\subsection{Analyses of Host Transcriptomics}

One-day-old B. longissima and O. nipae pupae were infected by $\mathrm{Tb}-\mathrm{Bl}$ and $\mathrm{Tb}-\mathrm{On}$, respectively. Pupae were harvested at $24 \mathrm{~h}$ post-parasitisation and non-parasitised pupae were synchronously collected as controls. A total of four libraries were constructed, including Bl-NP, Bl-PP, On-NP and On-PP. For each library, three biological replicates were required and at least seven pupae were included in each biological replicate. Total RNA samples were isolated by an Eastep ${ }^{\circledR}$ Super total RNA extraction kit (Promega, Fitchburg, WI, USA) according to the manufacturer's protocol and sent to Majorbio ${ }^{\circledR}$ company (Shanghai, China) for sequencing and analysis. The construction and sequencing of a total of four cDNA libraries was implemented on an Illumina Hiseq 2500 platform with paired-end 
reads of $125 \mathrm{bp}$. Subsequent analysis of the transcriptomes was performed similar to the previous protocols [86]. In brief, the clean reads were assembled using Trinity [87] and output as unigenes. Unigenes were then annotated by BLASTx with an E-value $\leq 1 \mathrm{e}^{-5}$ against the NCBI non-redundant (NR), Swiss-Prot, Cluster of Orthologous Groups (COG), Gene ontology (GO) and Kyoto Encyclopedia of Genes and Genome (KEGG) protein databases. Protein domains were annotated with the Pfam 26.0 database (November 2011, 13,672 families) using the PfamScan software [88]. The relative abundance of unigenes was output as TPM (Transcripts Per Million reads) values by RSEM software. Differentially expressed genes (DEGs) between non-parasitised and parasitised host pupae were identified on the basis of $\log _{2}$ fold $\geq 0.585$ (fold change $\geq 1.5$ ) and $q$-value $<0.05$ (corrected $p$-value with Benjamini/Hochberg test using DESeq2), and then subjected to GO functional and KEGG pathway enrichment analyses using Fisher's exact test. Only GO terms or biological pathways with the adjusted $p$-value $<0.05$ was considered as statistically significantly enriched.

For orthologue analysis in two species, O. nipae unigenes with high-quality were applied to construct a local blast database and B. longissima unigenes were utilised for blast alignment with $70 \%$ coverage, $70 \%$ identity and E-value $\leq 1 \mathrm{e}^{-5}$. In turn, $B$. longissima unigenes were set as a local blast database and $O$. nipae unigenes were used to do blast alignment with the same parameter settings. Finally, O. nipae unique genes and B. longissima unique genes were subjected to GO and KEGG function analyses.

\subsection{Statistical Analysis}

One-way ANOVA and Tukey's multiple comparisons test $(p<0.05)$ was performed to analyse the data of variation of host THCs at different time points after injection of virulence factors/PBS, encapsulation index and PO activity, with the homogeneity of variance. Welch's / Brown-Forsythe ANOVA and Dunnett's T3 multiple comparisons test was used to reveal the influence of virulence on host THCs at $24 \mathrm{~h}$ post injection. Unpaired $t$-test $(p<0.05)$ was used to determine significant differences of THCs between the two hosts. The above statistical analyses were conducted using GraphPad Prism 8.0.2 for Windows. The DHC ratio and phagocytosis ratio among treatments were analysed by $\mathrm{R} \times \mathrm{C}$ chi-square test at statistical significance of $p<0.01$ using SPSS 22.0 (IBM Corp. Armonk, NY, USA).

\section{Conclusions}

The venom and ovarian fluids of T. brontispae contribute to the success of parasitism on hosts, and the venom is essential for adaptability to a new host. The venom of T. brontispae unloaded weapons-granulocytes to inhibit host immunity and decompose/degrade the host fat body, having a stronger virulence in the case of Tb-Onvenom. Thus, T. brontispae generated stress-induced evolutionary adaptations in O. nipae, which made O. nipae a perfect alternative host. T. brontispae performed well when encountering local versus nonlocal hosts, suggesting that the parasitoid wasp has good evolutionary plasticity and that the theory of local adaptation does not apply to this system. The lack of local adaptation can be explained by a number of reasons due to the complexity of the traits involved in the host-parasitoid interactions and/or strategies used by T. brontispae. Our findings suggest that the venom undergoes rapid evolution when facing different hosts, which helps to expand the host range. We presume that the T. brontispae-B. longissima/O. nipae systems are useful models for understanding of the evolutionary processes of the parasitoid wasps targeting different hosts with variable physiological dimensions, illustrating the trade-off mechanisms in parasitoid strategies. A better understanding of the functions of venom composition, host immune response traits, hormone system, nutrition metabolism and participating genes is required to further investigate the mechanism of stress-induced evolution and trade-off in the parasitoid wasp and host system. 
Supplementary Materials: The following are available online at https://www.mdpi.com/article/10 $.3390 /$ ijms22073581/s1.

Author Contributions: Conceptualization, Y.-M.H., B.-Z.T. and H.-J.Z.; methodology and validation, H.-J.Z. and B.-Z.T.; investigations, H.-J.Z., Y.-P.L., H.-Y.L., R.W., L.F. and Q.-C.J.; resources, Y.-M.H. and B.-Z.T.; writing—original draft preparation, H.-J.Z.; writing—review and editing, B.-Z.T.; supervision, B.-Z.T. and Y.-M.H. All authors have read and agreed to the published version of the manuscript.

Funding: This research was supported by grants from the National Natural Science Foundation of China (31672086 and 31471829), the National Key R\&D Program of China (2017YFC1200600) and the Scientific Research Foundation of Graduate School of Fujian Agriculture and Forestry University (324-1122yb053).

Institutional Review Board Statement: Not applicable.

Informed Consent Statement: Not applicable.

Data Availability Statement: The data that support the findings of this study are available from the corresponding author upon reasonable request.

Acknowledgments: The authors would like to thank Zhengqiang Peng for providing the Tb-Bl strain of T. brontispae.

Conflicts of Interest: The authors declare no conflict of interest. The funders had no role in the design of the study; in the collection, analyses, or interpretation of data; in the writing of the manuscript, or in the decision to publish the results.

\section{References}

1. LaSalle, J.; Gauld, I.D. Parasitic hymenoptera and the biodiversity crisis. Redia 1991, 74, 315-334.

2. Godfray, H.C.J. Parasitoids, Behavioral and Evolutionary Ecology; Princeton University Press: Princeton, NJ, USA, $1994 ;$ pp. 1-38.

3. Pennacchio, F.; Strand, M.R. Evolution of developmental strategies in parasitic hymenoptera. Annu. Rev. Entomol. 2006, 51, 233-258. [CrossRef]

4. Beckage, N.E.; Gelman, D.B. Wasp parasitoid disruption of host development: Implications for new biologically based strategies for insect control. Annu. Rev. Entomol. 2004, 49, 299-330. [CrossRef]

5. Adamo, S.A.; Kovalko, I.; Turnbull, K.F.; Easy, R.H.; Miles, C.I. The parasitic wasp Cotesia congregata uses multiple mechanisms to control host (Manduca sexta) behaviour. J. Exp. Biol. 2016, 219, 3750-3758. [CrossRef]

6. Godfray, H.C.J. Host resistance, parasitoid virulence, and population dynamics. In Parasitoid Population Dynamics; Hochberg, M.E., Ives, A.R., Eds.; Princeton University Press: Princeton, NJ, USA, 2000; pp. 120-138.

7. Godfray, H.C.J.; Shimada, M. Parasitoids: A model system to answer questions in behavioral, evolutionary and population ecology. Res. Popul. Ecol. 1999, 41, 3-10. [CrossRef]

8. Thompson, J.N. Coevolution: The geographic mosaic of coevolutionary arms races. Curr. Biol. 2005, 15, 992-994. [CrossRef]

9. Fors, L.; Markus, R.; Theopold, U.; Ericson, L.; Hamback, P.A. Geographic variation and trade-offs in parasitoid virulence. J. Anim. Ecol. 2016, 85, 1595-1604. [CrossRef] [PubMed]

10. Strand, M.R.; Pech, L.L. Immunological basis for compatibility of parasite-host relationship. Annu. Rev. Entomol. 1995, 40, 31-56. [CrossRef]

11. Glatz, R.V.; Asgari, S.; Schmidt, O. Evolution of polydnaviruses as insect immune suppressors. Trends Microbiol. 2004, 12, 545-554. [CrossRef] [PubMed]

12. Labrosse, C.; Carton, Y.; Dubuffet, A.; Drezen, J.M.; Poirié, M. Active suppression of Drosophila melanogaster immune response by long gland products of the parasitic wasp Leptopilina boulardi. J. Insect Physiol. 2003, 49, 513-522. [CrossRef]

13. Carton, Y.; Poirié, M.; Nappi, A.J. Insect immune resistance to parasitoids. Insect Sci. 2008, 15, 67-87. [CrossRef]

14. Poirié, M.; Carton, Y.; Dubuffet, A. Virulence strategies in parasitoid Hymenoptera as an example of adaptive diversity. CR Biol. 2009, 32, 311-320. [CrossRef]

15. Asgari, S.; Rivers, D.B. Venom proteins from endoparasitoid wasps and their role in host-parasite interactions. Annu. Rev. Entomol. 2011, 56, 313-335. [CrossRef] [PubMed]

16. Burke, G.R.; Strand, M.R. Systematic analysis of a wasp parasitism arsenal. Mol. Ecol. 2014, 23, 890-901. [CrossRef] [PubMed]

17. Davies, D.H.; Vinson, S.B. Passive evasion by eggs of braconid parasitoid Cardiochiles nigriceps of encapsulation in vitro by haemocytes of host Heliothis virescens. Possible role for fibrous layer in immunity. J. Insect Physiol. 1986, 32, 1003-1010. [CrossRef]

18. $\mathrm{Hu}, \mathrm{J} . ; \mathrm{Yu}, \mathrm{X}$.; Fu, W.; Zhang, W. A Helix pomatia lectin binding protein on the extraembryonic membrane of the polyembryonic wasp Macrocentrus cingulum protects embryos from being encapsulated by hemocytes of host Ostrinia furnaclis. Dev. Comp. Immunol 2008, 32, 356-364. [CrossRef] [PubMed]

19. Bai, S.F.; Li, X.; Tang, L.Q.; Chen, X.X. Immunosuppressive factors carried by eggs of the parasitoid wasp Cotesia vestalis (Hymenoptera: Braconidae). Acta Entomol. Sin. 2009, 52, 487-494. 
20. Dorémus, T.; Jouan, V.; Urbach, S.; Cousserans, F.; Wincker, P.; Ravallec, M.; Wajnberg, E.; Volkoff, A.N. Hyposoter didymator uses a combination of passive and active strategies to escape from the Spodoptera frugiperda cellular immune response. J. Insect Physiol. 2013, 59, 500-508. [CrossRef]

21. Meng, E.; Qiao, T.; Tang, B.; Hou, Y.; Yu, W.; Chen, Z. Effects of ovarian fluid, venom and egg surface characteristics of Tetrastichus brontispae (Hymenoptera: Eulophidae) on the immune response of Octodonta nipae (Coleoptera: Chrysomelidae). J. Insect Physiol. 2018, 109, 125-137. [CrossRef] [PubMed]

22. Lively, C.M.; Dybdahl, M.F. Parasite adaptation to locally common host genotypes. Nature 2000, 405, 679-681. [CrossRef]

23. Dupas, S.; Boscaro, M. Geographic variation and evolution of immunosuppressive genes in a Drosophila parasitoid. Ecography 1999, 22, 284-291. [CrossRef]

24. Benelli, G.; Messing, R.H.; Wright, M.G.; Giunti, G.; Kavallieratos, N.G.; Canale, A. Cues triggering mating and host-seeking behavior in the aphid parasitoid Aphidius colemani (Hymenoptera: Braconidae: Aphidiinae): Implications for biological control. J. Econ. Entomol. 2014, 107, 2005-2022. [CrossRef]

25. Borzoui, E.; Naseri, B.; Mohammadzadeh-Bidarani, M. Adaptation of Habrobracon hebetor (Hymenoptera: Braconidae) to rearing on Ephestia kuehniella (Lepidoptera: Pyralidae) and Helicoverpa armigera (Lepidoptera: Noctuidae). J. Insect Sci. 2016, 16, 1-7.

26. Fors, L.; Mozuraitis, R.; Blazyte-Cereskiene, L.; Verschut, T.A.; Hamback, P.A. Selection by parasitoid females among closely related hosts based on volatiles: Identifying relevant chemical cues. Ecol. Evol. 2018, 8, 3219-3228. [CrossRef] [PubMed]

27. Konopka, J.K.; Poinapen, D.; Gariepy, T.; McNeil, J.N. Understanding the mismatch between behaviour and development in a novel host-parasitoid association. Sci. Rep. 2018, 48, 15677. [CrossRef] [PubMed]

28. Antolin, M.F.; Bjorksten, T.A.; Vaughn, T.T. Host-related fitness trade-offs in a presumed generalist parasitoid, Diaeretiella rapae (Hymenoptera: Aphidiidae). Ecol. Entomol. 2006, 31, 242-254. [CrossRef]

29. Fors, L.; Markus, R.; Theopold, U.; Hamback, P.A. Differences in cellular immune competence explain parasitoid resistance for two coleopteran species. PLoS ONE 2014, 9, e108795. [CrossRef] [PubMed]

30. Hopper, K.R.; Oppenheim, S.J.; Kuhn, K.L.; Lanier, K.; Hoelmer, K.A.; Heimpel, G.E.; Heraty, J.M. Counties not countries: Variation in host specificity among populations of an aphid parasitoid. Evol. Appl. 2019, 12, 815-829. [CrossRef]

31. Agrawal, A.; Lively, C.M. Infection genetics: Gene-for-gene versus matching-alleles models and all points in between. Evol. Ecol. Res. 2002, 4, 79-90.

32. Carton, Y.; Nappi, A.; Poirié, M. Genetics of anti-parasite resistance in invertebrates. Dev. Comp. Immunol. 2005, 29, 9-32. [CrossRef]

33. Dubuffet, A.; Dupas, S.; Frey, F.; Drezen, J.M.; Poirié, M.; Carton, Y. Genetic interactions between the parasitoid wasp Leptopilina boulardi and its Drosophila hosts. Heredity 2007, 98, 21-27. [CrossRef]

34. Dubuffet, A.; Doury, G.; Labrousse, C.; Drezen, J.M.; Carton, Y.; Poirié, M. Variation of success of Leptopilina boulardi in Drosophila yakuba: The mechanisms explored. Dev. Comp. Immunol. 2008, 32, 597-602. [CrossRef]

35. Dani, M.P.; Edwards, J.P.; Richards, E.H. Hydrolase activity in the venom of the pupal endoparasitic wasp, Pimpla hypochondriaca. Comp. Biochem. Physiol. B 2005, 141, 373-381. [CrossRef]

36. Siebert, A.L.; Wright, J.; Martinson, E.; Wheeler, D.; Werren, J.H. Parasitoid Venom induces metabolic cascades in fly hosts. Metabolomics 2015, 11, 350-366.

37. Harvey, J.A.; Malcicka, M. Nutritional integration between insect hosts and koinobiont parasitoids in an evolutionary framework. Entomol. Exp. Appl. 2016, 159, 181-188. [CrossRef]

38. Colinet, D.; Dubuffet, A.; Cazes, D.; Moreau, S.; Drezen, J.M.; Poirie, M. A serpin from the parasitoid wasp Leptopilina boulardi targets the Drosophila phenoloxidase cascade. Dev. Comp. Immunol. 2009, 33, 681-689. [CrossRef]

39. Er, A.; Sak, O.; Ergin, E.; Uçkan, F.; Rivers, D.B. Venom-induced immunosuppression: An overview of hemocyte-mediated responses. Psyche A J. Entomol. 2011, 21, 1-14. [CrossRef]

40. Wan, L.; Fang, Q.; Qian, C.; Wang, F.; Yu, X.Q.; Ye, G. Inhibition of host cell encapsulation through inhibiting immune gene expression by the parasitic wasp venom calreticulin. Insect Biochem. Mol. Biol. 2013, 43, 936-946.

41. Paredes, J.C.; Herren, J.K.; Schupfer, F.; Lemaitre, B. The role of lipid competition for endosymbiont-mediated protection against parasitoid wasps in Drosophila. Microbiology 2016, 7, 8. [CrossRef] [PubMed]

42. Visser, B.; Willett, D.S.; Harvey, J.A.; Alborn, H.T. Concurrence in the ability for lipid synthesis between life stages in insects. $R$. Soc. Open Sci. 2017, 4, 160815. [CrossRef] [PubMed]

43. Casewell, N.R.; Wuster, W.; Vonk, F.J.; Harrison, R.A.; Fry, B.G. Complex cocktails: The evolutionary novelty of venoms. Trends Ecol. Evol. 2013, 28, 219-229. [CrossRef]

44. Alvarado, G.; Holland, S.R.; DePerez-Rasmussen, J.; Jarvis, B.A.; Telander, T.; Wagner, N.; Mortimer, N.T. Bioinformatic analysis suggests potential mechanisms underlying parasitoid venom evolution and function. Genomics 2019, 12, 1096-1104. [CrossRef]

45. Cavigliasso, F.; Mathe-Hubert, H.; Kremmer, L.; Rebuf, C.; Gatti, J.L.; Malausa, T.; Poirie, M. Rapid and differential evolution of the venom composition of a parasitoid wasp depending on the host strain. Toxins 2019, 11, 629. [CrossRef]

46. Yan, Z.; Fang, Q.; Wang, L.; Liu, J.; Zhu, Y.; Wang, F.; Ye, G. Insights into the venom composition and evolution of an endoparasitoid wasp by combining proteomic and transcriptomic analyses. Sci. Rep. 2016, 6, e19604. [CrossRef]

47. Martinson, E.O.; Kelkar, Y.D.; Chang, C.H.; Werren, J.H. The evolution of venom by co-option of single-copy genes. Curr. Biol. 2017, 27, 2007-2013. [CrossRef] 
48. Senji Laxme, R.R.; Suranse, V.; Sunagar, K. Arthropod venoms: Biochemistry, ecology and evolution. Toxicon 2019, 158, 84-103. [CrossRef]

49. Chen, Q.; Peng, Z.Q.; Xu, C.N.; Tang, C.; Lu, B.Q.; Jin, Q.; Wan, F.H. Biological assessment of Tetrastichus brontispae, a pupal parasitoid of coconut leaf beetle Brontispa longissima. Biocontrol. Sci. Technol. 2010, 20, 283-295. [CrossRef]

50. Tang, B.Z.; Xu, L.; Hou, Y.M. Effects of rearing conditions on the parasitism of Tetrastichus brontispae on its pupal host Octodonta nipae. Biocontrol 2014, 59, 647-657. [CrossRef]

51. Tang, B.Z.; Meng, E.; Zhang, H.J.; Zhang, X.M.; Asgari, S.; Lin, Y.P.; Hou, Y.M. Combination of label-free quantitative proteomics and transcriptomics reveals intraspecific venom variation between the two strains of Tetrastichus brontispae, a parasitoid of two invasive beetles. J. Proteomics 2019, 192, 37-53. [CrossRef]

52. Richards, E.H.; Parkinson, N.M. Venom from the endoparasitic wasp Pimpla hypochondriaca adversely affects the morphology, viability, and immune function of hemocytes from larvae of the tomato moth, Lacanobia oleracea. J. Invert. Pathol. 2000, 76, 33-42. [CrossRef] [PubMed]

53. Kaeslin, M.; Reinhard, M.; Buhler, D.; Roth, T.; Pfister-Wilhelm, R.; Lanzrein, B. Venom of the egg-larval parasitoid Chelonus inanitus is a complex mixture and has multiple biological effects. J. Insect Physiol. 2010, 56, 686-694. [CrossRef]

54. Furihata, S.X.; Matsumoto, H.; Kimura, M.T.; Hayakawa, Y. Venom components of Asobara japonica impair cellular immune responses of host Drosophila melanogaster. Arch. Insect Biochem. Physiol. 2013, 83, 86-100. [CrossRef]

55. Pennacchio, F.; Caccia, S.; Digilio, M.C. Host regulation and nutritional exploitation by parasitic wasps. Curr. Opin Insect. Sci. 2014, 6, 74-79. [CrossRef]

56. Er, A.; Uçkan, F.; Rivers, D.B.; Ergİn, E.; Sak, O. Effects of parasitization and envenomation by the endoparasitic wasp Pimpla turionellae (Hymenoptera: Ichneumonidae) on hemocyte numbers, morphology, and viability of its host Galleria mellonella (Lepidoptera: Pyralidae). Annu. Entomol. Soc. Am. 2010, 103, 273-282. [CrossRef]

57. Falabella, P.; Riviello, L.; Pascale, M.; Lelio, I.D.; Tettamanti, G.; Grimaldi, A.; Pennacchio, F. Functional amyloids in insect immune response. Insect Biochem. Mol. 2012, 42, 203-211. [CrossRef]

58. Grimaldi, A.; Tettamanti, G.; Congiu, T.; Girardello, R.; Malagoli, D.; Falabella, P.; de Eguileor, M. The main actors involved in parasitization of Heliothis virescens larva. Cell Tissue Res. 2012, 350, 491-502. [CrossRef]

59. Zhai, X.; Zhao, X.F. Participation of haemocytes in fat body degradation via cathepsin L expression. Insect Mol. 2012, 21, 521-534. [CrossRef] [PubMed]

60. Richards, E.H.; Dani, M.P.; Bradish, H. Immunosuppressive properties of a protein (rVPr1) from the venom of the endoparasitic wasp, Pimpla hypochondriaca: Mechanism of action and potential use for improving biological control strategies. J. Insect Physiol. 2013, 59, 213-222. [CrossRef] [PubMed]

61. Wiegand, C.; Levin, D.; Gillespie, J.P.; Willott, E.; Kanost, M.R.; Trenczek, T. Monoclonal antibody M13 identifies a plasmatocyte membrane protein and inhibits encapsulation and spreading reactions of Manduca sexta hemocytes. Arch. Insect Biochem. Physiol. 2000, 45, 95-108. [CrossRef]

62. Williams, M.J.; Wiklund, M.L.; Wikman, S.; Hultmark, D. Rac1 signalling in the Drosophila larval cellular immune response. J. Cell Sci. 2006, 119, 2015-2024. [CrossRef] [PubMed]

63. Lang, A.E.; Schmidt, G.; Schlosser, A.; Hey, T.D.; Larrinua, I.M.; Sheets, J.J. Photorhabdus luminescens toxins adp-ribosylate actin and rhoa to force actin clustering. Science 2010, 327, 1139-1142. [CrossRef]

64. Ashida, M.; Brey, P.T. Recent advances in research on the insect prophenoloxidase cascade. In Molecular Mechanisms of Immune Responses in Insects; Brey, P.T., Hultmark, D., Eds.; Chapman \& Hall: London, UK, 1998; pp. 135-172.

65. Ling, E.J.; Yu, X.Q. Prophenoloxidase binds to the surface of hemocytes and is involved in hemocyte melanization in Manduca sexta. Insect Biochem. Mol. 2005, 35, 1356-1366. [CrossRef]

66. Ribeiro, C.; Brehelin, M. Insect haemocytes: What type of cell is that? J. Insect Physiol. 2006, 52, 417-429. [CrossRef] [PubMed]

67. Dudzic, J.P.; Shu, K.; Ueda, R.; Bergman, C.M.; Lemaitre, B. Drosophila innate immunity: Regional and functional specialization of prophenoloxidases. BMC Biol. 2015, 13, 81. [CrossRef]

68. Kato, Y.; Yoshida, T.; Miura, K.; Tanaka, T.; Ochiai, M. A novel type of hemocytes localizing melanization with high-spreading behavior in Mythimna separata. Arch. Insect Biochem. Physiol. 2014, 86, 221-239. [CrossRef] [PubMed]

69. Meng, E.; Tang, B.; Hou, Y.; Chen, X.; Chen, J.; Yu, X.Q. Altered immune function of Octodonta nipae (Maulik) to its pupal endoparasitoid, Tetrastichus brontispae Ferriere. Comp. Biochem. Physiol. B 2016, 198, 100-109. [CrossRef] [PubMed]

70. Li, Y.; Lu, J.F.; Feng, C.J.; Ke, X.; Fu, W.J. Role of venom and ovarian proteins in immune suppression of Ostrinia furnacalis (Lepidoptera: Pyralidae) larvae parasitized by Macrocentrus cingulum (Hymenoptera: Braconidae), a polyembryonic parasitoid. Insect Sci. 2007, 14, 93-100. [CrossRef]

71. Mabiala-Moundoungou, A.D.; Doury, G.; Eslin, P.; Cherqui, A.; Prevost, G. Deadly venom of Asobara japonica parasitoid needs ovarian antidote to regulate host physiology. J. Insect Physiol. 2010, 56, 35-41. [CrossRef]

72. Nalini, M.; Ibrahim, A.M.A.; Hwang, I.; Kim, Y. Altered actin polymerization of Plutella xylostella (L.) in response to ovarian calyx components of an endoparasitoid Cotesia plutellae (Kurdjumov). Physiol. Entomol. 2009, 34, 110-118. [CrossRef]

73. Eslin, P.; Prévost, G. Hemocyte load and immune resistance to Asobara tabida are correlated in species of the Drosophila melanogaster subgroup. J. Insect Physiol. 1998, 44, 807-816. [CrossRef]

74. Kraaijeveld, A.R.; Godfray, H.C.J. Evolution of host resistance and parasitoid counter-resistance. Adv. Parasit. 2009, 70, 257-280. 
75. Badyaev, A.V. Role of stress in evolution: From individual adaptability to evolutionary adaptation. Variation 2005, 277-302. [CrossRef]

76. Schluter, D. Evidence for ecological speciation and its alternative. Science 2009, 3, 737-741. [CrossRef] [PubMed]

77. Van Nouhuys, S.; Niemikapee, S.; Hanski, I. Variation in a host-parasitoid interaction across independent populations. Insects 2012, 3, 1236-1256. [CrossRef] [PubMed]

78. Sandrock, C.; Gouskov, A.; Vorburger, C. Ample genetic variation but no evidence for genotype specificity in an allparthenogenetic host-parasitoid interaction. J. Evol. Biol. 2010, 23, 578-585. [CrossRef] [PubMed]

79. Dubuffet, A.; Colinet, D.; Anselme, C.; Dupas, S.; Carton, Y.; Poirié, M. Variation of Leptopilina boulardi Success in Drosophila Hosts. Adv. Parasit. 2009, 70, 147-188.

80. Li, J.L.; Zhang, X.; Hou, Y.M.; Tang, B.Z. Effects of multiple mating on the fecundity of an invasive pest (Octodonta nipae): The existence of an intermediate optimal female mating rate. Physiol. Entomol. 2014, 39, 348-354. [CrossRef]

81. Zhang, H.J.; Lin, Y.P.; Liu, M.; Liang, X.Y.; Ji, Y.N.; Tang, B.Z.; Hou, Y.M. Functional conservation and division of two singlecarbohydrate-recognition domain C-type lectins from the nipa palm hispid beetle Octodonta nipae (Maulik). Dev. Comp. Immunol. 2019, 100, 103416. [CrossRef]

82. Zhang, H.J.; Tang, B.Z.; Lin, Y.P.; Chen, Z.M.; Zhang, X.M.; Ji, T.L.; Hou, Y.M. Identification of three prophenoloxidaseactivating factors (PPAFs) from an invasive beetle Octodonta nipae Maulik (Coleoptera: Chrysomelidae) and their roles in the prophenoloxidase activation. Arch. Insect Biochem. Physiol. 2017, 96, e21425. [CrossRef]

83. Zhang, X.M.; Zhang, H.J.; Liu, M.; Liu, B.; Zhang, X.F.; Ma, C.J.; Tang, B.Z. Cloning and immunosuppressive properties of an acyl-activating enzyme from the venom apparatus of Tetrastichus brontispae (Hymenoptera: Eulophidae). Toxins 2019, 11, 672. [CrossRef]

84. Koopman, R.; Schaart, G.; Hesselink, M.K. Optimisation of oil red O staining permits combination with immunofluorescence and automated quantification of lipids. Histochem. Cell Biol. 2001, 116, 63-68. [CrossRef] [PubMed]

85. Caccia, S.; Grimaldi, A.; Casartelli, M.; Falabella, P.; de Eguileor, M.; Pennacchio, F.; Giordana, B. Functional analysis of a fatty acid binding protein produced by Aphidius ervi teratocytes. J. Insect Physiol. 2012, 58, 621-627. [CrossRef] [PubMed]

86. Tang, B.Z.; Chen, J.; Hou, Y.M.; Meng, E. Transcriptome immune analysis of the invasive beetle Octodonta nipae (Maulik) (Coleoptera: Chrysomelidae) parasitized by Tetrastichus brontispae Ferriére (Hymenoptera: Eulophidae). PLoS ONE 2014, 9, e91482. [CrossRef] [PubMed]

87. Grabherr, M.G.; Haas, B.J.; Yassour, M.; Levin, J.Z.; Thompson, D.A.; Amit, I.; Regev, A. Full-length transcriptome assembly from RNA-Seq data without a reference genome. Nat. Biotechnol. 2011, 29, 644-652. [CrossRef] [PubMed]

88. Punta, M.; Coggill, P.C.; Eberhardt, R.Y.; Mistry, J.; Tate, J.; Boursnell, C.; Finn, R.D. The Pfam protein families database. Nucleic Acids Res. 2012, 36, D290-D301. [CrossRef] [PubMed] 